\title{
6. TRACE AND RARE EARTH ELEMENT CHEMISTRY OF VOLCANIC ASHES FROM SITES 918 AND 919: IMPLICATIONS FOR ICELANDIC VOLCANISM ${ }^{1}$
}

\author{
Peter D. Clift ${ }^{2}$ and J. Godfrey Fitton ${ }^{3}$
}

\begin{abstract}
Miocene to Holocene sediments cored at Sites 918 and 919 in the Irminger Basin contain windblown ash layers derived from Icelandic volcanoes. They represent the most explosive volcanism of the rift system. In contrast, only those eruptions that produced lava flowing far from the rift zone are now exposed on Iceland due to subsidence close to the rift axis. Sediments older than 3.2 Ma contain little fresh glass, due to the hydrating effect of diagenetic fluids, although one basaltic layer dated at 10.5 Ma and three rhyolitic layers dated at 12.1-12.7 Ma were found to contain fresh glass. Major, trace, and rare earth element data have been collected from aphyric glass shards from these ash layers, using a combination of electron and ion microprobe technology. The ashes are dominantly basaltic and tholeiitic in character and are principally derived from the rift zone, with minor input from off-axis sources, probably the Snaefellsnes Peninsula. Their trace element characteristics are consistent with derivation from a mantle source similar to that below Iceland today, although the degree of this enrichment, as modeled by $\mathrm{Zr}$ / $\mathrm{Nb}$, can be seen to be variable over short periods of time and to have greater range than that found in exposed Icelandic lavas of the same age. The variations are not attributable to fractional crystallization. The ashes indicate that either different volcanoes were erupting compositionally variable lavas at any one time or that the composition varied rapidly with time, or both. These variations are homogenized by magma mixing prior to eruption in the large volume flows preserved in the Tertiary lava flows exposed in Iceland. Chemical variation seen in the ash layers may be due to variation in the degree of mantle melting, mantle heterogeneity, or derivation of melt from different depths within the melting column. Melting is thought to have commenced within a garnet-bearing mantle and continued in a spinel-bearing zone.
\end{abstract}

\section{INTRODUCTION}

Ocean Drilling Program (ODP) Leg 152 drilled a transect of holes across the continent-ocean transition offshore East Greenland along a latitude of approximately $63^{\circ} \mathrm{N}$. One of the principal objectives of the cruise was to examine the nature of the regional volcanism and to chart the evolution of the Icelandic mantle plume, which is thought to be responsible for the enhanced topography and volcanism on modern Iceland, compared with the rest of the Mid-Atlantic Ridge. During the breakup of the northeast Atlantic, the Iceland Plume may have contributed heat to the asthenosphere under the rift, so producing the thick sequences of lavas imaged as the seaward-dipping reflector series (SDRS). The recognition of a strong thermal input in the form of the thick lava sequences and absence of any chemically enriched components in the lavas (Shipboard Scientific Party, 1994a; 1994b) suggest that there may have been a marked detachment between the thermal and chemical effects of the plume at the time of continental breakup. A similar effect can be seen today, as the effects of the modern plume extend as far south as $62^{\circ} \mathrm{N}$ on the Reykjanes Ridge in terms of trace and rare earth elements and radiogenic isotope ratios (Hart et al., 1973; Schilling, 1973). However, elevated topography on the spreading ridge extends as far south as $50^{\circ} \mathrm{N}$ (Klein and Langmuir, 1987), as well as north along the Kolbeinsey Ridge (Mertz et al., 1991), indicating that the thermal effect of the plume is still more widespread than the chemical. The goal of this paper is to investigate the chemical character and evolution of the plume. Key questions addressed were (1) to what extent is the modern volcanism in Iceland representative of past activity in terms of its chemistry and (2) has the chemistry of the plume undergone any systematic changes and if so, over what time scales do these changes occur. We attempt

${ }^{1}$ Saunders, A.D., Larsen, H.C., and Wise, S.W., Jr. (Eds.), 1998. Proc. ODP, Sci. Results, 152: College Station, TX (Ocean Drilling Program).

${ }^{2}$ Department of Geology and Geophysics, Woods Hole Oceanographic Institution, Woods Hole, MA 02543, U.S.A. pclift@whoi.edu

${ }^{3}$ Department of Geology and Geophysics, Grant Institute, University of Edinburgh, West Mains Road, Edinburgh, EH9 3JW, United Kingdom. to address some of these problems mostly by examining the volcanic ash record recovered at Sites 918 and 919 in the Irminger Basin (Fig. 1). A few analyses were also made from the Paleogene at Site 914 on the East Greenland Shelf. The Greenland Shelf sites were not examined for Neogene ashes because this area was covered and strongly eroded by glaciers during this time (Larsen, Saunders, Clift, et al., 1994). In each case the ash material was analyzed using ion and electron microprobes and the chemistry examined to eliminate non-Icelandic volcanic centers as sources of the ash.

\section{ICELANDIC VOLCANISM}

The nature of recent volcanism in Iceland has been extensively investigated and compared with the older basalts exposed over east and west Iceland and dating back into the middle Miocene (approximately $16 \mathrm{Ma}$ ) in the northwest of the island (Hemond et al., 1993; Hardarson and Fitton, 1994). Except for the lone alkalic center at Snaefellsnes, modern volcanism on Iceland is concentrated in the eastern and western rift zones, representing the onshore continuation of the Mid-Atlantic Ridge. Physically, the Pleistocene volcanoes differ from the Tertiary basalts due to their eruption under the ice sheets that then covered the island. Nonetheless, extensive evidence exists for frequent explosive volcanism on Iceland (e.g., Sigurdsson and Loebner, 1981; Schmincke et al., 1982), making it the major source of airfall ashes in the Northeast Atlantic. The volcanic rocks of the neovolcanic rift zone differ from older Tertiary flows in terms of their geochemical variability. Although the vast majority of the modern volcanic rocks are basalts, the trace element chemistry shows that the degree of enrichment of the lavas with respect to incompatible elements is variable. An incompatible element is one that is not easily incorporated into the crystal lattices of mantle minerals. Therefore, when a given piece of mantle melts, the liquid produced will have more of any given incompatible element than of other more compatible elements when compared to the original mantle source. In this paper we employ $\mathrm{Zr} / \mathrm{Nb}$ to model the relative enrichment of magmas in high field strength elements, $\mathrm{Zr}$ being a more compatible high field strength element than $\mathrm{Nb}$. Both elements are readily measured and 


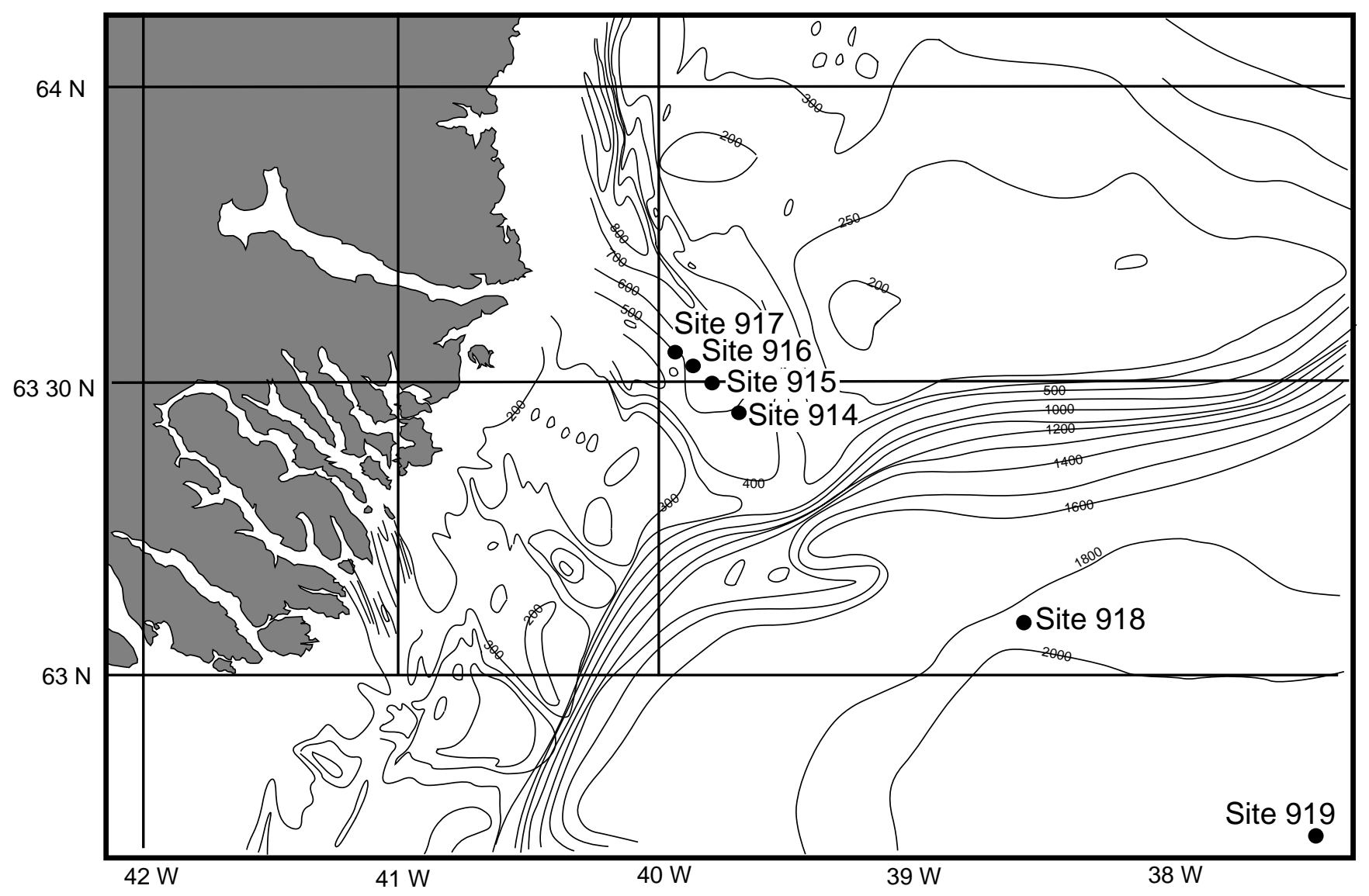

Figure 1. Map of the East Greenland Margin and Irminger Basin showing the location of the Leg 152 drill sites (Larsen, Saunders, Clift, et al., 1994).

the errors obtained at the concentrations encountered in Iceland lavas are small, giving high confidence in the results.

Hardarson and Fitton (1994) demonstrated that the Tertiary lavas outside the neovolcanic zone have $\mathrm{Zr} / \mathrm{Nb}$ values ranging from 6 to 13 , whereas recent volcanism in the neovolcanic zone shows a much wider spread, with values up to 34 . The reason for the spread in values may be related to the nature of crust formation in Iceland, rather than reflecting anomalous volcanism in the present day. The most widely accepted model for crustal formation is that of Pálmason (1981; 1986), who proposed a spreading mechanism for the midocean ridge on Iceland, featuring strong subsidence of material erupted in the rift zone (Fig. 2). As such, the rocks exposed at the surface over much of Iceland will only represent those flows that were able to travel far from the central rift zone. These flows will typically be the largest flows and may not be representative of the diversity of magmatism at any one time. For the largest flows, small-scale chemical heterogeneity will tend to be eliminated by mixing in large magma chambers. The variability in the modern rift zone would suggest that the exposed Tertiary lavas only show the lower values of $\mathrm{Zr} / \mathrm{Nb}$. To sample rocks with rarer high values of $\mathrm{Zr} / \mathrm{Nb}$, the melt needs to be removed from the mantle and erupted with a minimum of mixing, a process that is unlikely in large magma chambers.

The ashes recovered at Sites 918 and 919 span an overlapping time period with the lavas of onshore Iceland and allow this model of spreading and chemical variability to be tested. To have reached the Irminger Basin, the ash must have been blown to the southwest following an explosive eruption. One obvious problem when attempting to see greater chemical variability in the ashes is that the largest eruptions could also have been the most explosive, in which case they will be chemically identical to the outcropping Tertiary lavas. Although large volume eruptions are often explosive, studies of Icelandic activity show that violent phreatomagmatic eruptions are not always ac- companied by massive outpourings of lavas (e.g., Surtsey; Walker and Croasdale, 1972).

\section{SEDIMENTOLOGY AND PETROGRAPHY}

Figure 3 shows the location within the sedimentary column of ashes analyzed during this study. All the Neogene grains analyzed in this study were removed from primary airfall ash layers recovered at Sites 918 and 919, whereas some of the Oligocene and Eocene grains were reworked and are present as particles in shallow marine sandstones. The Neogene ashes are typically interbedded with sequences of clays and siliciclastic silts, interpreted as distal turbidites or the glacially deposited material dumped from melting glacier fronts and icebergs. This is especially true in the late Miocene-Holocene section of Site $918(0.0-543.5$ meters below seafloor [mbsf]). There is no evidence to suggest major reworking of the ash layers as turbidites, although disruption and particle dispersion by bioturbation is common. Ashes at Site 919, located closer to Iceland, are as much as $3 \mathrm{~cm}$ thick, whereas those layers at Site 918 are typically thinner and are often largely dispersed by burrowing. Where bioturbation was intense, the presence of ash particles was only revealed by microscopic smear slide study because there was no perceptible banding in the core itself. Nevertheless, it is reasonable to assume that these disseminated particles are representative of the volcanism at the time of deposition of the background sediment. Bioturbation only distributes the sediment particles locally, usually within $20 \mathrm{~cm}$ of the original layer, so that the overall stratigraphic progression is maintained. Thin ash layers (Fig. 4) show minor disruption by bioturbation. Undisturbed layers are sharp-based and show a grading in grain size and color up into hemipelagic sediments. Although slumping is known from the ocean islands of the Pacific (e.g., Hawaii; Garcia and Hull, 1994), a 

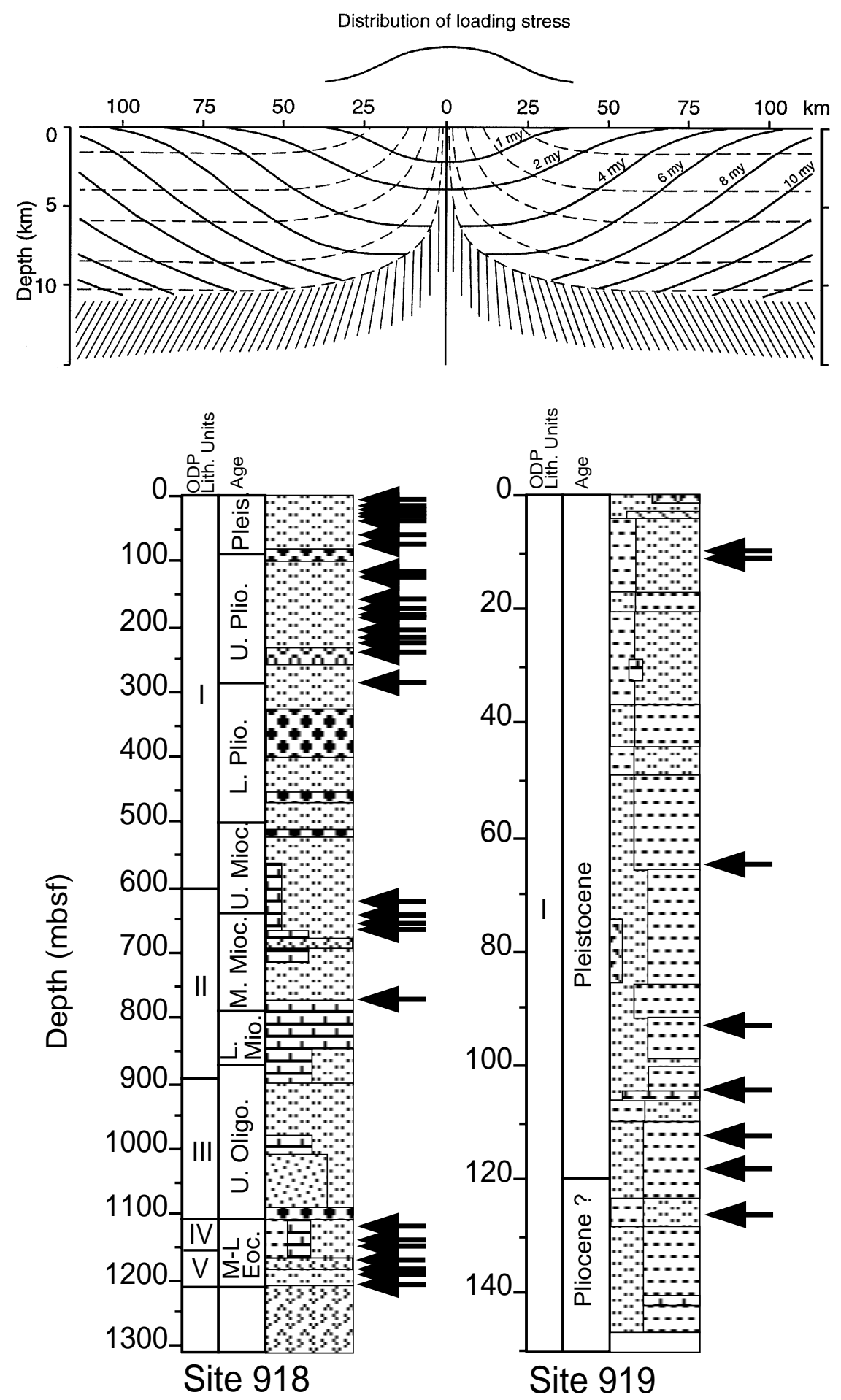

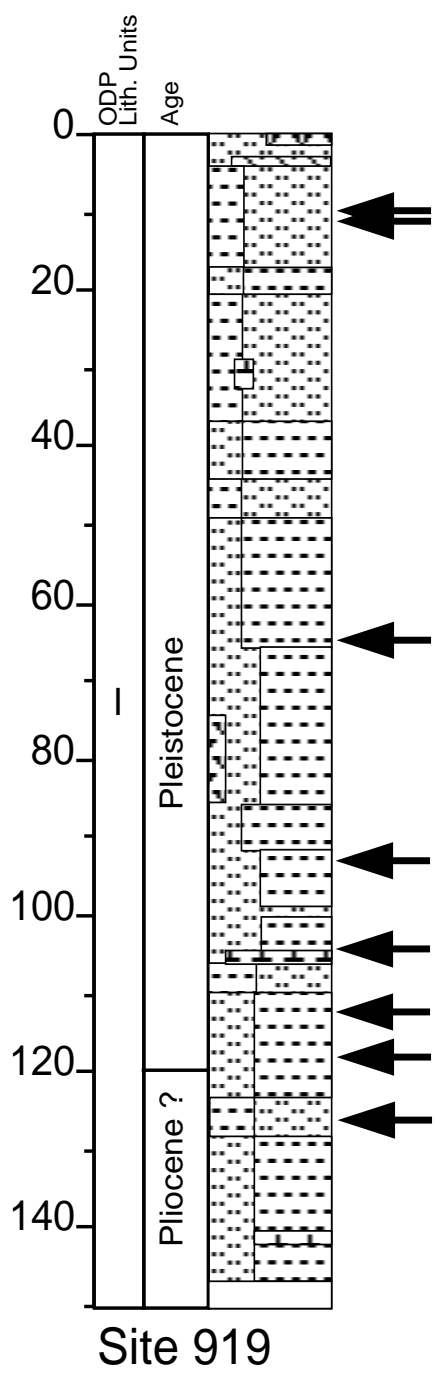

$\begin{array}{ll} & \text { Clay or claystone } \\ \text { 3. Silt or siltstone } \\ 8 \%\end{array}$
$\$ 4$
Gravel or conglomerate
.
Nannofossil ooze or chalk
Volcanic ash
\%. Basaltic lavas

Figure 2. Schematic diagram of Icelandic spreading model, showing the subsidence of lavas in the rift zone and the exposure of only the most distal of the older flows at the surface (Pálmason, 1981).
Figure 3. Schematic sedimentary logs for the sections cored at Sites 918 and 919. Arrows indicate level from which ash material was removed for analysis. 


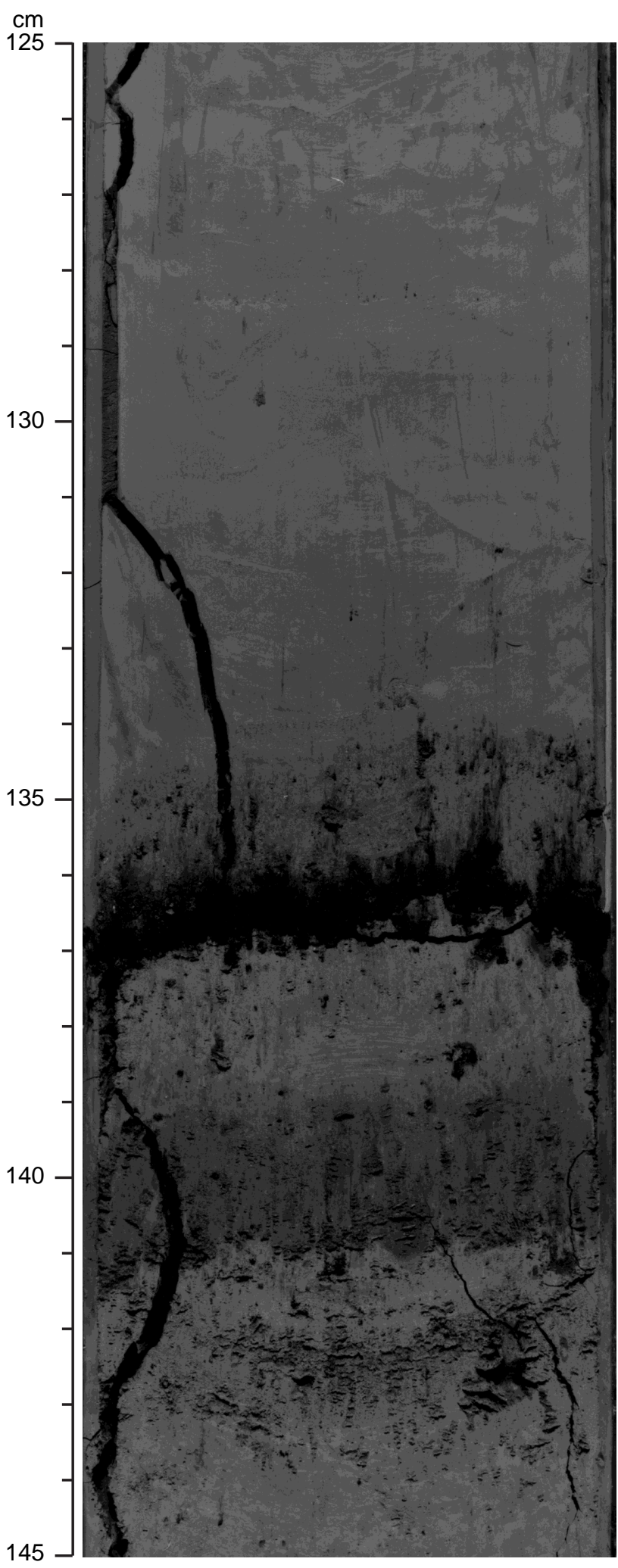

Figure 4. Representative example of distinct ash bed from Site 919. turbidite origin for the layers recovered during Leg 152 can be rejected, as no evidence exists to suggest erosion or redeposition by current activity (c.f. Bouma, 1962).

The vast majority of the undisturbed ash layers are dark colored, suggestive of a basaltic chemistry. Smear-slide examination indicates that the ash is dominated by volcanic glass shards that are very angular in form and usually show vesicles testifying to the volcanic origin. Up to $95 \%$ of grains within individual ash layers are glass, with the remainder made up by accessory minerals, principally feldspars and pyroxenes.

The amount of fresh glass and the number of individual ash layers decreases very markedly below 290 mbsf at Site 918 . Between 530 and $650 \mathrm{mbsf}$, the calcium content of the pore waters reaches a maximum and the magnesium content is at a minimum (Shipboard Scientific Party, 1994b). The chemistry within this zone is thought to be due to the alteration of volcanic glass. Although this, and the presence of palagonitized glass within the sediment, may explain the paucity of glass below $530 \mathrm{mbsf}$, it does not explain why there is so little present between 290 and 530 mbsf. There is not a clear explanation for such a drop off in material. Donn and Ninokovich (1980) suggest that there was a maximum in Icelandic volcanism during the Pliocene, as recorded at a number of drill sites throughout the northeast Atlantic. In contrast, Sigurdsson and Loebner (1981) used ashes recovered north of Iceland during Deep Sea Drilling Project (DSDP) Leg 38 to suggest a maximum in volcanism during the PliocenePleistocene. Both of these studies were disputed by Bitschene et al., (1989), who used the better recovery from ODP Leg 104 cores on the Vøring Plateau to infer no maximum in volcanism. However, exactly how good a Pliocene-Pleistocene record this area provides is open to question because it was covered by pack ice. If Icelandic volcanism really did peak during the Pliocene-Pleistocene, then other factors, such as climatic conditions, must have prevented the deposition of ash on the East Greenland Shelf, as the first appearance of abundant ash layers at Sites 919 and 918 is later than the 5.0 Ma date noted from northeast of Iceland (Sigurdsson and Loebner, 1981). Moberly and Campbell (1984) suggested that volcanism from the Hawaiian hot spot might be more intense during periods of normal geomagnetic polarity. Figure 5 shows the compiled data set for Sites 918 and 919 (discussed below) together with the geomagnetic reversal history of Cande and Kent (1992). It can be seen that although there is a correspondence between normal polarity and ashes from $0-1.5 \mathrm{Ma}$, this does not appear to be the case prior to that time. Even for the period 0-1.5 Ma, the small number of analyses makes this data set of questionable statistical significance. The ashes at Sites 918 and 919 do not support this model of episodic hot-spot magmatism.

The prevailing wind systems may have an influence on the accumulation of volcanic ash in the Irminger Basin. It is possible that the winds after 3.2 Ma were more prone to blowing material toward the southwest than had previously been the case. It is noteworthy that this time only shortly predates the onset of intense North Atlantic glaciation (Shackleton et al., 1984), and was most probably a period of significant climatic change, as global cooling intensified.

Interestingly, the position of the East Greenland drill sites is upwind of Iceland with respect to the dominant wind direction of the modern northeast Atlantic (Kennett, 1981). Stratospheric wind directions in the northeast Atlantic blow towards the east-northeast. Major explosive eruption columns may reach over $40 \mathrm{~km}$ into the atmosphere (i.e., well within the normal stratospheric range of $15-50 \mathrm{~km}$ ). In Iceland during this century, eruptions from Hekla resulted in columns that reached $17 \mathrm{~km}$ high in 1971 and $25 \mathrm{~km}$ high in 1947. Thus the accumulation of much ash in this region would be unexpected, which may explain the relatively modest amount of material found even as far east as Site 919. In comparison with DSDP drill sites northeast of Iceland (Donn and Ninkovich, 1980; Sigurdsson and Loebner, 1981; Bitschene et al., 1989; Desprairies et al., 1989), the thickness of individual ash beds near East Greenland is small. The material that accumulated at the Leg 152 drill sites must therefore represent either the products of exceptionally violent Plinean erup- 
A

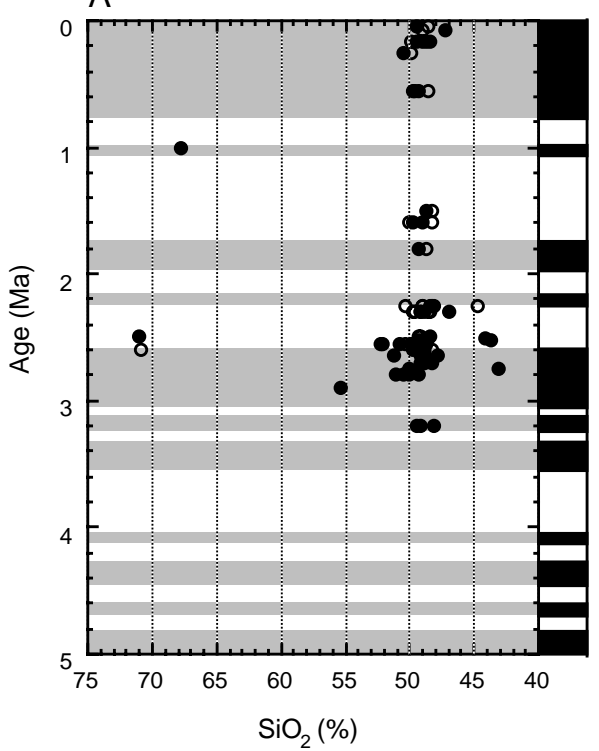

B

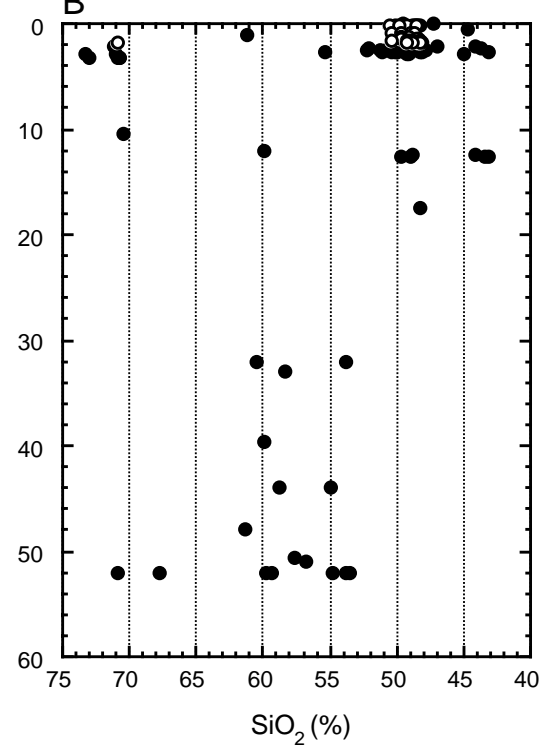

Figure 5. Variation in total silica content of glass grains with time since (A) 5.0 Ma and (B) $60 \mathrm{Ma}$ from Sites 918 (solid circles) and 919 (open circles). Geomagnetic reversal history from Cande and Kent (1992). tions or eruptions that took place during periods of abnormal wind direction. In either case, the Leg 152 ash material may not be representative of the full spectrum of Icelandic volcanism. Nevertheless, the ashes do provide valuable snapshots of the Icelandic activity, especially during the 0.0-3.2 Ma period.

\section{Paleogene Volcaniclastics}

A few analyses were made from the older parts of the section at Site 918. Oligocene sands, interpreted as turbidites, yielded a number of glass fragments whose alteration was not too severe. In these sediments, resedimentation has clearly occurred, which leaves their provenance using sedimentary data alone open to question. Their basaltic character means that they could represent airfall ashes of Oligocene age, derived from a proto-Iceland to the north. Alternatively, their derivation from onshore Greenland remains a possibility. If so, either the rift-related basalts of the seaward dipping sequences or late stage volcanism at Kangerlussuaq are possible sources. Although eroded away today, basalts are presumed to have been exposed onshore at $63^{\circ} \mathrm{N}$ during the Paleogene. The vast majority of the grains composing these sediments show clear evidence for derivation from the Greenland craton (Saito, this volume). A metamorphic source terrain is indicated by the presence of polycrystalline quartz, pyroxenes, amphiboles, and micas.

A small number of volcanic glass fragments were extracted from lower Eocene sediments directly overlying the weathered top of basalts at Site 918 . These sediments are strongly dominated by volcanic detritus and are of a shallow marine facies (Shipboard Scientific Party, 1994b). The sediments are reworked and, in view of their proximity to the basalts, were probably derived by erosion of the underlying basement, although additional input by airfall from volcanoes in the active rift, which at that time would have been close by and probably subaerial, remains a possibility.

\section{Dating of Ash Layers}

The age of a single ash layer and its constituent grains is determined using a combination of the shipboard biostratigraphic and magnetostratigraphic data (Shipboard Scientific Party, 1994b; 1994c). These data provide a number of age-control points on the section and yielded a stratigraphic age. A numerical age is then assigned from the time scale of Cande and Kent (1992). Between control points, the age at any one point is calculated assuming linear rates of sedimentation. Although probably inaccurate in detail, this method provides a good estimate of the age, and when plotted in sequence any age progression in the volcanism will not be affected.

\section{SAMPLE COLLECTION AND PREPARATION}

Samples were chosen from discrete volcaniclastic layers and were preferentially taken from the coarser grained bases of the layer where this was possible. Sediments were disaggregated by being mixed with water and placed in an ultrasonic bath for $24 \mathrm{hr}$. In the case of more indurated sediments, the sandstones and siltstones were treated with dilute $\mathrm{HCl}$ and then $\mathrm{NaOH}$ for $24 \mathrm{hr}$ each on a hotplate, agitating with a mechanical stirrer where necessary. After this the sediments were sieved through $125-\mu \mathrm{m}, 63-\mu \mathrm{m}$, and $45-\mu \mathrm{m}$ mesh and washed by a high-pressure water jet. A selection of grains from the largest size fraction $(>125 \mu \mathrm{m})$ was then mounted in epoxy resin and polished, using first $6-\mu \mathrm{m}$ and then $1-\mu \mathrm{m}$ diamond paste. The largest grain sizes were chosen due to the operational constraints of the ion microprobe. To make a single analysis, the beam of the probe covers a spot approximately $30 \mu \mathrm{m}$ across. Large grains were chosen to enhance the likelihood of being able to find such a spot for analysis. The slides were carbon coated before electron-probe analysis.

\section{ANALYTICAL PROCEDURES}

The samples were analyzed using the Cameca $S X 50$ electron microprobe at Texas A\&M University for $\mathrm{Si}, \mathrm{Al}, \mathrm{Na}, \mathrm{K}, \mathrm{Ca}, \mathrm{Mg}, \mathrm{Fe}, \mathrm{P}$, $\mathrm{Ti}, \mathrm{Mn}$, and, in some cases, Cr. A beam current of $10 \mathrm{nA}$ was employed, operating at a voltage of $20 \mathrm{kV}$. A count time of $30 \mathrm{~s}$ was used for each element.

Samples of basaltic and rhyolitic glass identified by the electron probe study were subsequently cleaned, coated in gold and analyzed using the Cameca ims $4 f$ ion microprobe at the University of Edinburgh, running Charles Evans and Associates software, for a suite of 20 trace and rare earth elements. The mafic glasses were chosen for trace element analysis to minimize the geochemical effect of fractional crystallization and thus yield the greatest amount of information on the petrogenetic history and source characteristics of the magmas. Electron backscattered images of glass obtained on the electron microprobe often showed the presence of microphenocrysts within the glass. The glass grains that were analyzed by the ion microprobe were either aphyric or contained the minimum number of phenocrysts within the available sample set. There is no way of establishing 
whether phenocryst phases were actually absent from the host magma of an aphyric glass fragment on eruption.

Representative major element chemical analyses for the volcanic glass grains probed are given in Table 1 . Trace and rare earth element data are shown in Table 2 . The reliability of the data is a key question. Very few of the Neogene grains show any visible cloudiness attributable to hydration. Alteration generally increases down section, a common phenomenon in marine ashes. The high surface area to volume ratio of ash shards, especially vesicular ones, promotes the hydration of the glass and results in a relatively short life span for small glass shards in the sedimentary column (Hay and Ijima, 1968). Alteration can be seen in the analyses as low analytical totals, although these can also arise from an indigenous volatile content in the melt (Burnham and Jahns, 1962). In general, however, the probe data from both sites show high totals, close to $100 \%$, suggesting that alteration has not affected these specimens to a significant degree. Lower totals, around $93 \mathrm{wt} \%$, are seen between 652.0 and $660.0 \mathrm{mbsf}$ at Site 918. This is mostly due to the greater age and degree of hydration of these specimens and to a lesser extent because the amount of water increases with silica content during fractionation.

An important additional source of error and uncertainty is the loss of sodium under the electron beam (Neilson and Sigurdsson, 1981). The expected levels of total alkalies at around $8 \mathrm{wt} \%$ in the most siliceous glasses are not achieved because sodium declines sharply with silica content from basaltic andesite compositions onwards. Potassium values are scattered but are not so conspicuously subject to loss.

Sodium loss under the beam causes the apparent concentration of other elements to rise. As sodium loss is related to silica content and is not subject to a constant loss factor and volatile content is also broadly related to silica, the two effects counteract each other and measured abundances of most elements, except sodium, are probably not far from their volatile-free levels. The data have therefore been left uncorrected. Instead, analyses with abnormally low totals have been excluded.

It should be noted that the trace element data obtained on the ion microprobe are not affected by effects analogous to sodium loss under the electron beam, although a correction would still be necessary to arrive at volatile-free values. This correction is likely to be very small, as Icelandic magmas are generally dry and thus even the acid glasses would not have been water saturated on eruption.

\section{Analytical Errors}

The precision and reproducibility of the trace element measurements are assumed to closely approximate to those given by the Poisson counting statistics for each element. This assumption is supported by earlier work on the same probe (Hinton and Upton, 1991) and on other basaltic glass grains (Clift and Dixon, 1994). In particular, the smoothness of the rare earth element (REE) plots obtained from the glasses suggest that the errors are not significantly greater than the uncertainties in the counting statistics, even at low concentrations $(<1.0 \mathrm{ppm})$. Chemically similar elements are seen to show the same trends within the data set, regardless of whether the element is a relatively abundant one (e.g., Zr) or present in low concentrations (e.g., $\mathrm{Ho}, \mathrm{Tb}$ ). Table 3 shows the count rate errors for a typical analysis from Site 918. It is noteworthy that for some geochemically important elements, such as $\mathrm{Nb}$, the ion probe method is able to achieve good precision ( $2 \%$ error), as is shown by the correlation between $\mathrm{Nb}$ and $\mathrm{Zr}$ on Figure 6. Clift and Dixon (1994) estimated that background interference became a statistical problem in measuring $\mathrm{Nb}$ below 0.4 ppm, well below the values being recorded here. The scatter on this plot is primarily due to real variations in the $\mathrm{Zr} / \mathrm{Nb}$ ratio.

\section{MAJOR ELEMENT CHEMISTRY}

Fresh glass appears to be confined to Site 919 and Site 918 above $290 \mathrm{mbsf}$. Below this level, glass is confined to a horizon at $619 \mathrm{mbsf}$ and three beds within an interval of 652-660 mbsf at Site 918. These beds contain only rhyolitic glasses. Of the Oligocene and Eocene grains analyzed, much of the material proved to be basaltic but was typically altered to smectite. However, the cores of a few grains, especially those from calcite-cemented layers, provided high total percentages of major elements, suggesting that alteration was not too advanced and that some useful information could be gained by the consideration of water immobile elements.

The total silica contents of the freshest 0.0-3.2 Ma glasses are displayed in Figure 5. The vast majority of the analyses show basaltic values, ranging from 47.0 to $52.5 \mathrm{wt} \% \mathrm{SiO}_{2}$. The majority of grains within this group show a more confined range of $48-51 \mathrm{wt} \% \mathrm{SiO}_{2}$, typical of Icelandic tholeiite. $\mathrm{MgO}$ is mostly high, typically $>6 \mathrm{wt} \%$, ranging as high as $7.8 \mathrm{wt} \%$, which suggests that the grains are not very evolved and thus appropriate for petrogenetic modeling. Figure 7 shows a triangular alkali-iron-magnesium (AFM) diagram (Irvine and Baragar, 1971). The analyzed grains clearly fall along the trend of a tholeiitic fractionation series. The major element ash data indicate that volcanism since 3.2 Ma has been dominantly basaltic and tholeiitic. Rare silicic eruptions are noted at 1.0, 1.8, and 2.5 Ma, as well as earlier at $12.1,12.5$, and $12.7 \mathrm{Ma}$. No temporal trend in the nature of the major element chemistry can be seen and effectively one basaltic ash layer is indistinguishable from another.

\section{TRACE AND RARE EARTH ELEMENT CHEMISTRY}

A group of 20 trace and rare earth elements was measured using an ion microprobe. The full analyses, together with the corresponding major element analyses, are shown in Table 2 . Table 3 shows a typical analysis from Sample 152-918A-8H-2, $34 \mathrm{~cm}$, with concentrations and percentage error determined from the counting statistics. This percentage varies with the total concentration of any given element and its ionization potential. In general, however, the errors are low, the vast majority under $5 \mathrm{wt} \%$. Notable exceptions are $\mathrm{Sm}$ and $\mathrm{Lu}$, which approach $10 \%$, whereas $\mathrm{U}$, present in only tiny quantities, records uncertainties of $31.6 \mathrm{wt} \%$. The results from the ion probe work are thus comparable, and in a number of instances superior to other analytical techniques such as inductively coupled plasma mass spectrometry (ICP-MS) or X-ray fluorescence (XRF). In this paper we shall consider both the overall chemical character of the glasses and individual elemental ratios. The degree of enrichment of incompatible elements over compatible elements is a key factor and for this purpose we looked at two elemental groups, the high field strength elements and the REE.

As mentioned above, the degree of alteration of the glasses younger than 3.2 Ma is considered to be low due to the general clarity of the glass under microscopic examination and the high analytical totals derived from electron probe analysis. Hydration, however, has affected older grains to a greater degree, resulting in totals less than $90 \%$. However, many trace and rare earth elements are considered to be immobile in water (Pearce, 1983). Provided the hydration has not advanced too far, these elements may still be useful for petrogenetic purposes.

The multi-element spider diagram is typically used to display the overall incompatible element characteristics of a rock (Thompson et al., 1983). In a spider diagram, the elements are ordered to give a smooth curve for average mid-ocean ridge basalts (MORB; Sun, 1980), which in effect means an increasing incompatibility of the elements in lherzolite during incipient partial melting from right to left. Figures 8 and 9 show spider diagrams for glasses of different ages at Sites 918 and 919, respectively. In each case, the total concentration of the elements has been normalized against a primitive mantle standard (Sun and McDonough, 1989). The patterns shown by all the Miocene-Pleistocene examples are typical of lavas derived from an undepleted mantle source. The flat or gently inclined patterns have a relative enrichment in the most incompatible elements (i.e., toward the left of the diagram), and contrast with natural 
Table 1. Representative major elemental compositions for individual glass grains as measured by electron probe.

\begin{tabular}{|c|c|c|c|c|c|c|c|c|c|c|c|c|c|c|}
\hline $\begin{array}{l}\text { Core, sample, } \\
\text { interval }(\mathrm{cm})\end{array}$ & $\begin{array}{l}\text { Depth } \\
\text { (mbsf) }\end{array}$ & $\begin{array}{l}\text { Age } \\
\text { (Ma) }\end{array}$ & $\begin{array}{c}\mathrm{SiO}_{2} \\
(\mathrm{wt} \%)\end{array}$ & $\begin{array}{l}\mathrm{TiO}_{2} \\
(\mathrm{wt} \%)\end{array}$ & $\begin{array}{l}\mathrm{Al}_{2} \mathrm{O}_{3} \\
(\mathrm{wt} \%)\end{array}$ & $\begin{array}{l}\mathrm{FeO} \\
(\mathrm{wt} \%)\end{array}$ & $\begin{array}{l}\mathrm{MnO} \\
(\mathrm{wt} \%)\end{array}$ & $\begin{array}{l}\mathrm{MgO} \\
(\mathrm{wt} \%)\end{array}$ & $\begin{array}{c}\mathrm{CaO} \\
(\mathrm{wt} \%)\end{array}$ & $\begin{array}{l}\mathrm{Na}_{2} \mathrm{O} \\
(\mathrm{wt} \%)\end{array}$ & $\begin{array}{l}\mathrm{K}_{2} \mathrm{O} \\
(\mathrm{wt} \%)\end{array}$ & $\begin{array}{l}\mathrm{Cr}_{2} \mathrm{O}_{3} \\
(\mathrm{wt} \%)\end{array}$ & $\begin{array}{l}\mathrm{P}_{2} \mathrm{O}_{5} \\
(\mathrm{wt} \%)\end{array}$ & $\begin{array}{l}\text { Total } \\
\text { (wt \%) }\end{array}$ \\
\hline $\begin{array}{r}152-918 \mathrm{~A}- \\
2 \mathrm{H}-2,34 \\
2 \mathrm{H}-4,24\end{array}$ & $\begin{array}{l}3.64 \\
6.54\end{array}$ & $\begin{array}{l}0.04 \\
0.08\end{array}$ & $\begin{array}{l}49.492 \\
47.203\end{array}$ & $\begin{array}{l}3.056 \\
2.470\end{array}$ & $\begin{array}{l}13.551 \\
14.736\end{array}$ & $\begin{array}{r}14.281 \\
9.645\end{array}$ & $\begin{array}{l}0.235 \\
0.195\end{array}$ & $\begin{array}{l}5.740 \\
7.795\end{array}$ & $\begin{array}{l}10.017 \\
12.616\end{array}$ & $\begin{array}{l}2.636 \\
2.176\end{array}$ & $\begin{array}{l}0.400 \\
0.849\end{array}$ & $\begin{array}{l}0.007 \\
0.030\end{array}$ & $\begin{array}{l}0.288 \\
0.347\end{array}$ & $\begin{array}{l}99.703 \\
98.062\end{array}$ \\
\hline $\begin{array}{c}152-919 \mathrm{~B}- \\
2 \mathrm{H}-1,43 \\
2 \mathrm{H}-1,43 \\
2 \mathrm{H}-1,106 \\
2 \mathrm{H}-1,106 \\
2 \mathrm{H}-1,106 \\
2 \mathrm{H}-1,106 \\
2 \mathrm{H}-1,106\end{array}$ & $\begin{array}{r}9.63 \\
9.63 \\
10.26 \\
10.26 \\
10.26 \\
10.26 \\
10.26\end{array}$ & $\begin{array}{l}0.14 \\
0.14 \\
0.15 \\
0.15 \\
0.15 \\
0.15 \\
0.15\end{array}$ & $\begin{array}{l}48.572 \\
49.087 \\
49.062 \\
49.066 \\
49.950 \\
49.840 \\
49.605\end{array}$ & $\begin{array}{l}3.067 \\
3.375 \\
2.457 \\
2.653 \\
2.526 \\
2.806 \\
2.591\end{array}$ & $\begin{array}{l}13.023 \\
12.886 \\
13.327 \\
12.914 \\
13.497 \\
13.052 \\
13.264\end{array}$ & $\begin{array}{l}13.988 \\
14.483 \\
13.717 \\
14.344 \\
13.055 \\
14.950 \\
13.116\end{array}$ & $\begin{array}{l}0.183 \\
0.265 \\
0.194 \\
0.243 \\
0.168 \\
0.310 \\
0.227\end{array}$ & $\begin{array}{l}5.509 \\
4.741 \\
5.903 \\
5.364 \\
5.820 \\
5.078 \\
6.205\end{array}$ & $\begin{array}{r}9.634 \\
9.269 \\
10.299 \\
9.665 \\
10.346 \\
9.767 \\
10.604\end{array}$ & $\begin{array}{l}2.677 \\
2.605 \\
2.529 \\
2.612 \\
2.682 \\
1.723 \\
2.498\end{array}$ & $\begin{array}{l}0.431 \\
0.578 \\
0.349 \\
0.426 \\
0.415 \\
0.423 \\
0.420\end{array}$ & $\begin{array}{l}0.009 \\
0.002 \\
0.063 \\
0.023 \\
0.011 \\
0.000 \\
0.000\end{array}$ & $\begin{array}{l}0.369 \\
0.473 \\
0.272 \\
0.357 \\
0.363 \\
0.343 \\
0.311\end{array}$ & $\begin{array}{l}97.462 \\
97.764 \\
98.172 \\
97.667 \\
98.833 \\
98.292 \\
98.841\end{array}$ \\
\hline $\begin{array}{c}152-918 \mathrm{~A}- \\
3 \mathrm{H}-3,142 \\
3 \mathrm{H}-3,142 \\
3 \mathrm{H}-3,142 \\
4 \mathrm{H}-2,48 \\
5 \mathrm{H}-3,103 \\
5 \mathrm{H}-3,103\end{array}$ & $\begin{array}{l}15.42 \\
15.42 \\
15.42 \\
22.78 \\
34.03 \\
34.03\end{array}$ & $\begin{array}{l}0.16 \\
0.16 \\
0.16 \\
0.26 \\
0.55 \\
0.55\end{array}$ & $\begin{array}{l}49.442 \\
48.469 \\
48.697 \\
50.573 \\
49.263 \\
49.756\end{array}$ & $\begin{array}{l}3.787 \\
2.803 \\
3.283 \\
1.927 \\
2.357 \\
2.233\end{array}$ & $\begin{array}{l}10.746 \\
14.059 \\
12.850 \\
13.239 \\
13.971 \\
13.808\end{array}$ & $\begin{array}{l}15.268 \\
12.267 \\
13.289 \\
13.890 \\
12.190 \\
12.325\end{array}$ & $\begin{array}{l}0.317 \\
0.207 \\
0.231 \\
0.244 \\
0.199 \\
0.208\end{array}$ & $\begin{array}{l}6.592 \\
5.970 \\
5.745 \\
6.031 \\
6.632 \\
6.721\end{array}$ & $\begin{array}{l}11.145 \\
10.304 \\
10.233 \\
10.168 \\
11.296 \\
11.250\end{array}$ & $\begin{array}{l}2.091 \\
2.565 \\
2.538 \\
2.284 \\
2.341 \\
2.396\end{array}$ & $\begin{array}{l}0.504 \\
0.546 \\
0.385 \\
0.232 \\
0.289 \\
0.270\end{array}$ & $\begin{array}{l}0.036 \\
0.070 \\
0.053 \\
0.033 \\
0.038 \\
0.023\end{array}$ & $\begin{array}{l}0.345 \\
0.380 \\
0.314 \\
0.263 \\
0.210 \\
0.204\end{array}$ & $\begin{array}{r}100.273 \\
97.640 \\
97.618 \\
98.884 \\
98.786 \\
99.194\end{array}$ \\
\hline $\begin{array}{r}\text { 152-919A- } \\
7 \mathrm{H}-6,135 \\
7 \mathrm{H}-6,135 \\
7 \mathrm{H}-6,135 \\
7 \mathrm{H}-6,135\end{array}$ & $\begin{array}{l}64.35 \\
64.35 \\
64.35 \\
64.35\end{array}$ & $\begin{array}{l}0.90 \\
0.90 \\
0.90 \\
0.90\end{array}$ & $\begin{array}{l}48.539 \\
48.298 \\
48.326 \\
50.048\end{array}$ & $\begin{array}{l}3.247 \\
3.470 \\
3.316 \\
1.247\end{array}$ & $\begin{array}{l}12.849 \\
12.916 \\
12.962 \\
14.518\end{array}$ & $\begin{array}{l}14.674 \\
14.627 \\
15.358 \\
10.837\end{array}$ & $\begin{array}{l}0.263 \\
0.215 \\
0.258 \\
0.206\end{array}$ & $\begin{array}{l}5.057 \\
5.095 \\
5.100 \\
7.748\end{array}$ & $\begin{array}{r}9.487 \\
9.434 \\
9.753 \\
12.829\end{array}$ & $\begin{array}{l}2.737 \\
2.726 \\
2.696 \\
2.201\end{array}$ & $\begin{array}{l}0.557 \\
0.572 \\
0.573 \\
0.155\end{array}$ & $\begin{array}{l}0.020 \\
0.004 \\
0.000 \\
0.049\end{array}$ & $\begin{array}{l}0.453 \\
0.343 \\
0.362 \\
0.156\end{array}$ & $\begin{array}{l}97.883 \\
97.700 \\
98.704 \\
99.994\end{array}$ \\
\hline $\begin{array}{c}152-918 \mathrm{~A}- \\
6 \mathrm{H}-1,122\end{array}$ & 41.02 & 1.00 & 67.722 & 0.042 & 18.638 & 1.902 & 0.024 & 0.870 & 0.383 & 8.244 & 2.087 & 0.000 & 0.000 & 99.912 \\
\hline $\begin{array}{c}152-919 \mathrm{~B}- \\
3 \mathrm{H}-3,85 \\
3 \mathrm{H}-3,85 \\
4 \mathrm{H}-3,115 \\
4 \mathrm{H}-3,115\end{array}$ & $\begin{array}{r}93.85 \\
93.85 \\
103.65 \\
103.65\end{array}$ & $\begin{array}{l}1.31 \\
1.31 \\
1.47 \\
1.47\end{array}$ & $\begin{array}{l}48.780 \\
48.968 \\
49.023 \\
50.422\end{array}$ & $\begin{array}{l}2.803 \\
2.431 \\
0.778 \\
1.395\end{array}$ & $\begin{array}{l}13.080 \\
12.196 \\
15.017 \\
14.463\end{array}$ & $\begin{array}{r}14.135 \\
14.126 \\
9.220 \\
11.204\end{array}$ & $\begin{array}{l}0.300 \\
0.257 \\
0.152 \\
0.208\end{array}$ & $\begin{array}{l}5.355 \\
7.354 \\
8.822 \\
7.253\end{array}$ & $\begin{array}{r}9.625 \\
10.240 \\
13.728 \\
11.486\end{array}$ & $\begin{array}{l}2.584 \\
1.821 \\
1.784 \\
2.225\end{array}$ & $\begin{array}{l}0.430 \\
0.380 \\
0.067 \\
0.261\end{array}$ & $\begin{array}{l}0.030 \\
0.011 \\
0.027 \\
0.046\end{array}$ & $\begin{array}{l}0.318 \\
0.266 \\
0.013 \\
0.111\end{array}$ & $\begin{array}{l}97.440 \\
98.050 \\
98.631 \\
99.074\end{array}$ \\
\hline $\begin{array}{r}152-918 \mathrm{~A}- \\
8 \mathrm{H}-2,34 \\
10 \mathrm{H}-3,2 \\
10 \mathrm{H}-3,2\end{array}$ & $\begin{array}{l}60.64 \\
80.82 \\
80.82\end{array}$ & $\begin{array}{l}1.50 \\
1.60 \\
1.60\end{array}$ & $\begin{array}{l}48.747 \\
49.721 \\
49.088\end{array}$ & $\begin{array}{l}3.072 \\
2.767 \\
2.379\end{array}$ & $\begin{array}{l}12.866 \\
13.260 \\
13.385\end{array}$ & $\begin{array}{l}13.950 \\
13.355 \\
12.953\end{array}$ & $\begin{array}{l}0.233 \\
0.201 \\
0.227\end{array}$ & $\begin{array}{l}5.310 \\
5.977 \\
5.917\end{array}$ & $\begin{array}{r}9.454 \\
10.102 \\
10.732\end{array}$ & $\begin{array}{l}2.586 \\
2.593 \\
2.493\end{array}$ & $\begin{array}{l}0.443 \\
0.404 \\
0.279\end{array}$ & $\begin{array}{l}0.003 \\
0.045 \\
0.031\end{array}$ & $\begin{array}{l}0.357 \\
0.195 \\
0.253\end{array}$ & $\begin{array}{l}97.021 \\
98.620 \\
97.737\end{array}$ \\
\hline $\begin{array}{r}152-919 \mathrm{~B}- \\
5 \mathrm{H}-3,39 \\
5 \mathrm{H}-3,99 \\
5 \mathrm{H}-3,99 \\
5 \mathrm{H}-3,99 \\
5 \mathrm{H}-3,99 \\
5 \mathrm{H}-3,99 \\
6 \mathrm{H}-1,43\end{array}$ & $\begin{array}{l}112.99 \\
112.99 \\
112.99 \\
112.99 \\
112.99 \\
112.99 \\
118.93\end{array}$ & $\begin{array}{l}1.60 \\
1.60 \\
1.60 \\
1.60 \\
1.60 \\
1.60 \\
1.69\end{array}$ & $\begin{array}{l}49.672 \\
49.721 \\
48.372 \\
48.555 \\
49.110 \\
49.291 \\
48.572\end{array}$ & $\begin{array}{l}3.363 \\
3.495 \\
3.507 \\
3.307 \\
3.327 \\
3.318 \\
3.067\end{array}$ & $\begin{array}{l}13.158 \\
13.626 \\
12.897 \\
12.879 \\
12.981 \\
13.607 \\
13.023\end{array}$ & $\begin{array}{l}15.085 \\
15.618 \\
15.007 \\
14.364 \\
14.059 \\
14.416 \\
13.988\end{array}$ & $\begin{array}{l}0.291 \\
0.209 \\
0.295 \\
0.226 \\
0.308 \\
0.183 \\
0.183\end{array}$ & $\begin{array}{l}5.029 \\
4.649 \\
5.072 \\
5.150 \\
5.354 \\
4.290 \\
5.509\end{array}$ & $\begin{array}{r}9.268 \\
8.997 \\
9.487 \\
9.340 \\
10.127 \\
9.109 \\
9.634\end{array}$ & $\begin{array}{l}2.491 \\
1.999 \\
2.729 \\
2.702 \\
2.444 \\
2.910 \\
2.677\end{array}$ & $\begin{array}{l}0.652 \\
0.607 \\
0.529 \\
0.563 \\
0.646 \\
0.587 \\
0.431\end{array}$ & $\begin{array}{l}0.000 \\
0.018 \\
0.000 \\
0.036 \\
0.031 \\
0.024 \\
0.009\end{array}$ & $\begin{array}{l}0.337 \\
0.389 \\
0.401 \\
0.511 \\
0.440 \\
0.460 \\
0.369\end{array}$ & $\begin{array}{l}99.346 \\
99.328 \\
98.296 \\
97.633 \\
98.827 \\
98.195 \\
97.462\end{array}$ \\
\hline $\begin{array}{r}152-919 \mathrm{~B}- \\
6 \mathrm{H}-1,43 \\
6 \mathrm{H}-6,87 \\
6 \mathrm{H}-6,87 \\
6 \mathrm{H}-6,87 \\
6 \mathrm{H}-6,87 \\
6 \mathrm{H}-6,87\end{array}$ & $\begin{array}{l}118.93 \\
126.87 \\
126.87 \\
126.87 \\
126.87 \\
126.87\end{array}$ & $\begin{array}{l}1.69 \\
1.77 \\
1.77 \\
1.77 \\
1.77 \\
1.77\end{array}$ & $\begin{array}{l}49.087 \\
48.893 \\
48.994 \\
48.842 \\
48.787 \\
50.404\end{array}$ & $\begin{array}{l}3.375 \\
2.927 \\
2.767 \\
2.579 \\
2.561 \\
2.603\end{array}$ & $\begin{array}{l}12.886 \\
13.155 \\
12.959 \\
13.025 \\
13.042 \\
13.253\end{array}$ & $\begin{array}{l}14.483 \\
14.142 \\
14.955 \\
14.007 \\
13.171 \\
13.034\end{array}$ & $\begin{array}{l}0.265 \\
0.257 \\
0.265 \\
0.257 \\
0.212 \\
0.266\end{array}$ & $\begin{array}{l}4.741 \\
5.457 \\
5.246 \\
5.425 \\
6.390 \\
5.368\end{array}$ & $\begin{array}{r}9.269 \\
9.635 \\
9.190 \\
9.849 \\
11.736 \\
9.345\end{array}$ & $\begin{array}{l}2.605 \\
2.749 \\
2.704 \\
2.730 \\
2.599 \\
2.802\end{array}$ & $\begin{array}{l}0.578 \\
0.397 \\
0.414 \\
0.391 \\
0.183 \\
0.545\end{array}$ & $\begin{array}{l}0.002 \\
0.000 \\
0.000 \\
0.033 \\
0.009 \\
0.000\end{array}$ & $\begin{array}{l}0.473 \\
0.266 \\
0.344 \\
0.266 \\
0.252 \\
0.351\end{array}$ & $\begin{array}{l}97.764 \\
97.878 \\
97.838 \\
97.404 \\
98.942 \\
97.971\end{array}$ \\
\hline $\begin{array}{c}152-918 \mathrm{~A}- \\
11 \mathrm{H}-2,31\end{array}$ & 89.11 & 1.80 & 49.314 & 2.076 & 13.948 & 11.525 & 0.227 & 6.795 & 11.089 & 2.490 & 0.303 & 0.000 & 0.182 & 97.949 \\
\hline $\begin{array}{r}152-919 \mathrm{~B}- \\
7 \mathrm{H}-1,37 \\
7 \mathrm{H}-1,37\end{array}$ & $\begin{array}{l}128.37 \\
128.37\end{array}$ & $\begin{array}{l}1.80 \\
1.80\end{array}$ & $\begin{array}{l}48.317 \\
70.894\end{array}$ & $\begin{array}{l}3.397 \\
0.226\end{array}$ & $\begin{array}{r}12.964 \\
7.827\end{array}$ & $\begin{array}{r}15.235 \\
6.389\end{array}$ & $\begin{array}{l}0.305 \\
0.281\end{array}$ & $\begin{array}{l}5.061 \\
0.019\end{array}$ & $\begin{array}{l}9.704 \\
0.275\end{array}$ & $\begin{array}{l}2.676 \\
4.027\end{array}$ & $\begin{array}{l}0.537 \\
2.811\end{array}$ & $\begin{array}{l}0.022 \\
0.047\end{array}$ & $\begin{array}{l}0.355 \\
0.007\end{array}$ & $\begin{array}{l}98.573 \\
92.803\end{array}$ \\
\hline $\begin{array}{c}\text { 152-918A- } \\
13 \mathrm{H}-6,35 \\
13 \mathrm{H}-6,35 \\
13 \mathrm{H}-6,35 \\
13 \mathrm{H}-6,35 \\
14 \mathrm{H}-3,0\end{array}$ & $\begin{array}{l}114.15 \\
114.15 \\
114.15 \\
114.15 \\
118.8\end{array}$ & $\begin{array}{l}2.25 \\
2.25 \\
2.25 \\
2.25 \\
2.30\end{array}$ & $\begin{array}{l}48.382 \\
48.286 \\
48.140 \\
48.226 \\
49.120\end{array}$ & $\begin{array}{l}2.107 \\
2.061 \\
1.878 \\
2.054 \\
2.408\end{array}$ & $\begin{array}{l}13.778 \\
13.820 \\
13.570 \\
13.551 \\
13.446\end{array}$ & $\begin{array}{l}11.900 \\
12.165 \\
11.965 \\
12.423 \\
13.060\end{array}$ & $\begin{array}{l}0.221 \\
0.173 \\
0.238 \\
0.242 \\
0.158\end{array}$ & $\begin{array}{l}6.936 \\
6.917 \\
6.942 \\
6.976 \\
6.230\end{array}$ & $\begin{array}{l}11.499 \\
11.719 \\
11.406 \\
11.366 \\
10.441\end{array}$ & $\begin{array}{l}2.474 \\
2.482 \\
2.376 \\
2.441 \\
2.524\end{array}$ & $\begin{array}{l}0.202 \\
0.176 \\
0.203 \\
0.243 \\
0.364\end{array}$ & $\begin{array}{l}0.044 \\
0.008 \\
0.013 \\
0.022 \\
0.018\end{array}$ & $\begin{array}{l}0.110 \\
0.201 \\
0.175 \\
0.253 \\
0.143\end{array}$ & $\begin{array}{l}97.653 \\
98.008 \\
96.906 \\
97.617 \\
97.912\end{array}$ \\
\hline
\end{tabular}


Table 1 (continued).

\begin{tabular}{|c|c|c|c|c|c|c|c|c|c|c|c|c|c|c|}
\hline $\begin{array}{l}\text { Core, sample, } \\
\text { interval (cm) }\end{array}$ & $\begin{array}{l}\text { Depth } \\
\text { (mbsf) }\end{array}$ & $\begin{array}{l}\text { Age } \\
\text { (Ma) }\end{array}$ & $\begin{array}{c}\mathrm{SiO}_{2} \\
\text { (wt\%) }\end{array}$ & $\begin{array}{l}\mathrm{TiO}_{2} \\
(\mathrm{wt} \%)\end{array}$ & $\begin{array}{l}\mathrm{Al}_{2} \mathrm{O}_{3} \\
(\mathrm{wt} \%)\end{array}$ & $\begin{array}{l}\mathrm{FeO} \\
\text { (wt\%) }\end{array}$ & $\begin{array}{l}\mathrm{MnO} \\
(\mathrm{wt} \%)\end{array}$ & $\begin{array}{l}\mathrm{MgO} \\
(\mathrm{wt} \%)\end{array}$ & $\begin{array}{c}\mathrm{CaO} \\
(\mathrm{wt} \%)\end{array}$ & $\begin{array}{l}\mathrm{Na}_{2} \mathrm{O} \\
(\mathrm{wt} \%)\end{array}$ & $\begin{array}{c}\mathrm{K}_{2} \mathrm{O} \\
(\mathrm{wt} \%)\end{array}$ & $\begin{array}{l}\mathrm{Cr}_{2} \mathrm{O}_{3} \\
(\mathrm{wt} \%)\end{array}$ & $\begin{array}{l}\mathrm{P}_{2} \mathrm{O}_{5} \\
(\mathrm{wt} \%)\end{array}$ & $\begin{array}{r}\text { Total } \\
(\mathrm{wt} \%)\end{array}$ \\
\hline $14 \mathrm{H}-3,0$ & 118.8 & 2.30 & 48.877 & 2.493 & 13.265 & 13.375 & 0.205 & 5.898 & 10.557 & 2.528 & 0.291 & 0.000 & 0.227 & 97.716 \\
\hline $14 \mathrm{H}-3,0$ & 118.8 & 2.30 & 49.230 & 1.805 & 14.220 & 11.184 & 0.158 & 7.280 & 11.934 & 2.306 & 0.283 & 0.050 & 0.124 & 98.574 \\
\hline $14 \mathrm{H}-3,0$ & 118.8 & 2.30 & 47.020 & 3.086 & 15.559 & 11.899 & 0.162 & 5.491 & 8.862 & 3.679 & 1.148 & 0.051 & 0.636 & 97.593 \\
\hline $18 \mathrm{H}-\mathrm{CC}, 14$ & 161.8 & 2.50 & 71.092 & 0.028 & 12.582 & 1.486 & 0.087 & 0.034 & $\begin{array}{l}0.002 \\
0.646\end{array}$ & 2.335 & $\begin{array}{l}1.140 \\
5.409\end{array}$ & $\begin{array}{l}0.000 \\
0.000\end{array}$ & $\begin{array}{l}0.000 \\
0.000\end{array}$ & 93.699 \\
\hline $18 \mathrm{H}-\mathrm{CC}, 14$ & $\begin{array}{l}161.8 \\
161.8\end{array}$ & 2.50 & 48.435 & 3.824 & $\begin{array}{l}12.582 \\
13.033\end{array}$ & $\begin{array}{l}1.480 \\
13.374\end{array}$ & $\begin{array}{l}0.087 \\
0.296\end{array}$ & $\begin{array}{l}0.034 \\
4.564\end{array}$ & $\begin{array}{l}0.040 \\
8.965\end{array}$ & 3.075 & $\begin{array}{l}1.476 \\
1.476\end{array}$ & $\begin{array}{l}0.000 \\
0.000\end{array}$ & $\begin{array}{l}0.000 \\
1.062\end{array}$ & 98.104 \\
\hline $20 \mathrm{H}-3,9$ & 174.39 & 2.52 & $\begin{array}{l}49.337 \\
4\end{array}$ & 1.375 & 15.247 & 10.317 & $\begin{array}{l}0.290 \\
0.188\end{array}$ & $\begin{array}{l}4.964 \\
7.985\end{array}$ & $\begin{array}{r}8.905 \\
12.051\end{array}$ & 2.118 & $\begin{array}{l}1.4 / 0 \\
0.408\end{array}$ & $\begin{array}{l}0.000 \\
0.056\end{array}$ & $\begin{array}{l}1.002 \\
0.098\end{array}$ & $\begin{array}{l}98.104 \\
99.180\end{array}$ \\
\hline $21 \mathrm{H}-2,109$ & 184.09 & 2.55 & 49.898 & 3.692 & 12.713 & 14.842 & 0.267 & 4.809 & 8.781 & 2.450 & 0.558 & 0.043 & 0.377 & 98.430 \\
\hline $21 \mathrm{H}-2,109$ & 184.09 & 2.55 & 52.061 & 3.565 & 12.550 & 13.914 & 0.331 & 3.625 & 7.708 & 2.993 & 0.793 & 0.048 & 0.508 & 98.096 \\
\hline $21 \mathrm{H}-2,109$ & 184.09 & 2.55 & 50.841 & 3.217 & 13.332 & $\begin{array}{l}13.614 \\
\end{array}$ & 0.204 & 4.876 & 8.954 & 2.783 & 0.648 & $\begin{array}{l}0.014 \\
0.014\end{array}$ & 0.397 & 98.880 \\
\hline $21 \mathrm{H}-2,109$ & 184.09 & 2.55 & 52.274 & 3.492 & 12.661 & 14.916 & 0.330 & 3.812 & 8.247 & 2.731 & 0.721 & 0.000 & 0.540 & 99.724 \\
\hline $23 \mathrm{X}-5,14$ & 205.94 & 2.60 & 49.765 & 3.247 & 12.904 & 14.830 & 0.234 & 4.990 & 9.328 & 2.783 & 0.512 & 0.000 & 0.240 & 98.833 \\
\hline $23 \mathrm{X}-5,14$ & 205.94 & 2.60 & 48.868 & 2.505 & 14.024 & 12.437 & 0.193 & 6.716 & 10.912 & 2.480 & 0.276 & 0.011 & 0.208 & 98.630 \\
\hline $23 \mathrm{X}-5,67$ & 206.47 & 2.63 & 48.940 & 2.242 & 13.641 & 13.599 & 0.204 & 6.680 & 10.848 & 2.305 & 0.248 & 0.055 & 0.221 & 98.983 \\
\hline $23 X-5,67$ & 206.47 & 2.63 & 49.034 & 2.355 & 13.784 & 13.225 & 0.255 & 6.654 & 10.756 & 2.365 & 0.274 & 0.028 & 0.221 & 98.951 \\
\hline $23 X-5,67$ & 206.47 & 2.63 & 49.096 & 2.408 & 13.904 & 13.123 & 0.160 & 6.932 & 10.776 & 2.352 & 0.227 & 0.051 & 0.279 & 99.308 \\
\hline $23 \mathrm{X}-5,67$ & 206.47 & 2.63 & 49.179 & 2.367 & 13.708 & 13.727 & 0.200 & 6.426 & 10.625 & 2.388 & 0.221 & 0.038 & 0.208 & 99.087 \\
\hline $23 \mathrm{X}-5,122$ & $\begin{array}{l}2007.02 \\
207.02\end{array}$ & 2.65 & 49.165 & 3.445 & 13.141 & 14.605 & 0.258 & $\begin{array}{l}0.420 \\
5.862\end{array}$ & 10.089 & $\begin{array}{l}2.500 \\
2.290\end{array}$ & 0.396 & $\begin{array}{l}0.000 \\
0.002\end{array}$ & $\begin{array}{l}0.434 \\
0.400\end{array}$ & 99.687 \\
\hline $23 \mathrm{X}-5,122$ & 207.02 & 2.65 & 47.875 & 1.960 & 15.501 & 12.652 & 0.224 & 7.835 & $\begin{array}{l}11.089 \\
\end{array}$ & 2.343 & 0.246 & 0.007 & 0.156 & 99.888 \\
\hline $23 \mathrm{X}-5,122$ & 207.02 & 2.65 & 51.239 & 2.767 & 13.165 & 13.657 & 0.261 & 4.447 & 8.710 & 2.746 & 0.703 & 0.000 & 0.489 & 98.184 \\
\hline $23 \mathrm{X}-5,122$ & 212.98 & 2.70 & 48.916 & 2.051 & 13.910 & 11.975 & $\begin{array}{l}0.201 \\
0.202\end{array}$ & $\begin{array}{l}4.441 \\
6.966\end{array}$ & $\begin{array}{l}0.110 \\
11.669\end{array}$ & 2.329 & 0.231 & 0.000 & $\begin{array}{l}0.409 \\
0.189\end{array}$ & 98.438 \\
\hline \multicolumn{15}{|l|}{ 152-918A- } \\
\hline $24 \mathrm{X}-3,128$ & 212.98 & 2.70 & 49.101 & 2.713 & 13.379 & 13.280 & 0.198 & 6.122 & 10.416 & 2.475 & 0.292 & 0.035 & 0.299 & 98.310 \\
\hline $24 \mathrm{X}-3,128$ & 212.98 & 2.70 & 48.256 & 3.178 & 13.138 & 14.903 & 0.217 & 5.631 & 10.443 & 2.672 & 0.346 & 0.000 & 0.388 & 99.172 \\
\hline $25 \mathrm{X}-4,109$ & 223.19 & 2.75 & 50.024 & 1.245 & 13.548 & 12.358 & 0.207 & 6.792 & 11.360 & 2.121 & 0.137 & 0.007 & 0.131 & 97.930 \\
\hline $25 \mathrm{X}-4,109$ & 223.19 & 2.75 & 43.104 & 1.339 & 12.917 & 14.923 & 0.219 & 11.577 & 10.823 & 1.234 & 0.968 & 0.029 & 0.000 & 97.133 \\
\hline $25 \mathrm{X}-7,4$ & 226.64 & 2.80 & 49.337 & 3.221 & 13.320 & 14.065 & 0.197 & 5.268 & 9.466 & 2.695 & 0.563 & 0.006 & 0.480 & 98.618 \\
\hline $25 X-7,4$ & 226.64 & 2.80 & 50.031 & 3.041 & 13.399 & 13.956 & 0.239 & 5.164 & 9.313 & 2.728 & 0.563 & 0.000 & $\begin{array}{l}0.400 \\
0.390\end{array}$ & 98.824 \\
\hline $25 \mathrm{X}-7,4$ & 226.64 & 2.80 & 50.434 & 3.162 & 13.427 & 13.572 & 0.195 & 5.267 & 9.337 & 2.732 & 0.559 & 0.050 & 0.436 & 99.171 \\
\hline $25 \mathrm{X}-7,4$ & 226.64 & 2.80 & 51.044 & 3.018 & 13.264 & 13.908 & 0.224 & 4.828 & 8.656 & 2.764 & 0.621 & 0.020 & 0.495 & 98.842 \\
\hline $28 \mathrm{X}-2,67$ & 246.27 & 2.90 & 55.398 & 0.122 & 27.815 & 1.412 & 0.000 & 0.292 & 10.567 & 5.194 & 0.348 & 0.000 & 0.020 & 101.168 \\
\hline $33 \mathrm{X}-2,59$ & 290.49 & 3.20 & 48.098 & 3.170 & 12.816 & 15.851 & 0.252 & 5.032 & 9.495 & 2.700 & 0.532 & 0.000 & 0.383 & 98.329 \\
\hline $33 \mathrm{X}-2,59$ & 290.49 & 3.20 & 49.446 & 1.751 & 13.748 & 12.835 & 0.221 & 6.501 & 11.329 & 2.423 & 0.345 & 0.015 & 0.157 & 98.771 \\
\hline $33 \times-2,59$ & 290.49 & 3.20 & 49.201 & 2.395 & 13.361 & 12.991 & 0.189 & 5.886 & 10.345 & 2.665 & 0.437 & 0.001 & 0.313 & 97.784 \\
\hline $33 \mathrm{X}-2,59$ & 290.49 & 3.20 & 49.116 & 2.319 & 13.813 & 12.409 & 0.226 & 6.596 & 11.496 & 2.531 & 0.331 & 0.025 & 0.208 & 99.070 \\
\hline \multicolumn{15}{|l|}{ 152-918D- } \\
\hline 36R-1, 17 & 619.07 & 10.50 & 45.012 & 0.402 & 12.451 & 14.356 & 0.223 & 11.153 & 12.155 & 1.039 & 0.738 & 0.061 & 0.000 & 97.590 \\
\hline $39 R-4,14$ & 652.54 & 12.10 & 73.278 & 0.065 & 11.965 & $\begin{array}{l}1.253 \\
1.253\end{array}$ & 0.101 & 0.169 & 1.151 & 2.923 & 2.900 & 0.024 & 0.000 & 93.829 \\
\hline $40 \mathrm{R}-2,36$ & 659.36 & 12.50 & 71.006 & 0.303 & 11.791 & 3.400 & 0.146 & 0.068 & 1.852 & 3.603 & 1.830 & 0.000 & 0.014 & 94.013 \\
\hline $40 \mathrm{R}-2,36$ & 659.36 & 12.50 & 70.738 & 0.194 & 11.712 & 3.560 & 0.072 & 0.093 & 1.905 & 3.597 & 1.791 & 0.026 & 0.034 & 93.722 \\
\hline $40 \mathrm{R}-3,114$ & 661.64 & 12.70 & 72.999 & 0.155 & 11.505 & 1.501 & 0.061 & 0.076 & 0.910 & 3.218 & 3.008 & 0.016 & 0.028 & 93.477 \\
\hline 40R-3, 114 & 661.64 & 12.70 & 70.758 & 0.263 & 11.909 & 3.614 & 0.137 & 0.116 & 1.787 & 3.343 & 1.980 & 0.010 & 0.048 & 93.965 \\
\hline 40R-3, 114 & 661.64 & 12.70 & 70.901 & 0.279 & 11.749 & 3.377 & 0.101 & 0.145 & 1.750 & 3.429 & 1.883 & 0.009 & 0.041 & 93.664 \\
\hline $40 \mathrm{R}-3,114$ & 661.64 & 12.70 & 70.502 & 0.279 & 11.898 & 3.384 & 0.117 & 0.148 & 1.754 & 3.154 & 1.964 & 0.000 & 0.014 & 93.214 \\
\hline $51 \mathrm{R}-6,67$ & 771.37 & 17.40 & 59.942 & 0.270 & 10.906 & 1.044 & 0.034 & 0.921 & 1.030 & 3.120 & 2.272 & 0.017 & 0.000 & 79.556 \\
\hline \multicolumn{15}{|l|}{ 152-914B- } \\
\hline $16 \mathrm{R}-1,90$ & 226.7 & 32.10 & 48.809 & 0.467 & 8.705 & 11.964 & 0.113 & 14.591 & 11.177 & 1.386 & 0.176 & 0.145 & 0.000 & 97.533 \\
\hline 16R-1, 90 & 226.7 & 32.10 & 44.187 & 1.570 & 12.306 & 13.485 & 0.179 & 11.881 & 11.397 & $\begin{array}{l}1.500 \\
1.543\end{array}$ & 0.926 & 0.127 & 0.046 & 97.647 \\
\hline $17 \mathrm{R}-1,146$ & 236.96 & 32.90 & 43.119 & 2.111 & 10.689 & 14.703 & 0.376 & 12.201 & 11.508 & 1.692 & 1.589 & 0.000 & 0.000 & 97.988 \\
\hline \multicolumn{15}{|l|}{ 152-918D- } \\
\hline $88 \mathrm{R}-1,42$ & 1118.22 & 39.70 & 53.759 & 0.336 & 9.671 & 11.777 & 0.111 & 6.840 & 0.194 & 0.558 & 2.804 & 0.015 & 0.107 & 86.172 \\
\hline $89 \mathrm{R}-4,49$ & 1132.39 & 44.04 & 54.750 & 0.967 & 12.368 & 10.643 & 0.007 & 3.493 & 0.490 & 0.383 & 3.987 & 0.039 & 0.321 & 87.448 \\
\hline $89 \mathrm{R}-4,49$ & 1132.39 & 44.04 & 59.404 & 0.768 & 7.335 & 12.798 & 0.000 & 2.782 & 0.229 & 0.229 & 4.646 & 0.013 & 0.113 & 88.317 \\
\hline $89 \mathrm{R}-4,49$ & 1132.39 & 44.04 & 59.800 & 0.760 & 7.649 & 12.397 & 0.020 & 2.751 & 0.230 & 0.242 & 4.432 & 0.000 & 0.167 & 88.448 \\
\hline $89 \mathrm{R}-4,49$ & 1132.39 & 44.04 & 70.839 & 0.473 & 3.506 & 5.275 & 0.007 & 1.049 & 0.296 & 0.286 & 1.523 & 0.000 & 0.110 & 83.364 \\
\hline $89 \mathrm{R}-4,49$ & 1132.39 & 44.04 & 59.861 & 1.241 & 8.154 & 7.392 & 0.070 & 2.064 & 0.292 & 0.331 & 2.564 & 0.031 & 0.312 & 82.312 \\
\hline $91 \mathrm{R}-2,10$ & 1148.1 & 47.90 & 53.499 & 3.886 & 14.083 & 7.483 & 0.048 & 2.712 & 0.634 & 0.479 & 1.153 & 0.010 & 0.067 & 84.054 \\
\hline $93 \mathrm{R}-2,64$ & $\begin{array}{l}1167.94 \\
1167.1\end{array}$ & 48.30 & 61.268 & 0.955 & $\begin{array}{r}4.010 \\
\end{array}$ & 10.304 & 0.000 & 1.747 & 0.123 & 0.636 & 3.060 & 0.000 & 0.364 & 82.867 \\
\hline $93 \mathrm{R}-2,64$ & 1167.94 & 48.30 & 61.358 & 1.623 & 10.384 & 7.021 & 0.002 & 3.301 & 0.158 & 1.205 & 1.795 & 0.076 & 0.238 & 87.161 \\
\hline \multicolumn{15}{|l|}{$152-918 \mathrm{D}-$} \\
\hline 95R-3, 94 & 1184.34 & 50.60 & 49.675 & 0.172 & 9.046 & 9.659 & 0.221 & 15.956 & 11.153 & 1.216 & 0.163 & 0.325 & 0.059 & 97.645 \\
\hline $96 \mathrm{R}-1,29$ & 1185.39 & 51.00 & 43.453 & 1.231 & 11.313 & 17.942 & 0.272 & 9.517 & 11.961 & 1.234 & 1.000 & 0.000 & 0.000 & 97.923 \\
\hline $96 \mathrm{R}-1,29$ & 1185.39 & 51.00 & 58.721 & 0.539 & 3.985 & 16.227 & 0.052 & 2.596 & 0.087 & 0.070 & 4.667 & 0.015 & 0.066 & 87.025 \\
\hline $96 \mathrm{R}-3,120$ & 1189.3 & 51.40 & 54.901 & 2.305 & 13.682 & 9.528 & 0.018 & 3.387 & 0.377 & 0.139 & 1.171 & 0.007 & 0.108 & 85.623 \\
\hline $100 \mathrm{R}-2,27$ & 1215.77 & 52.00 & 49.034 & 0.234 & 14.082 & 17.583 & 0.052 & 2.843 & 0.786 & 0.454 & 2.493 & 0.028 & 0.040 & 87.629 \\
\hline
\end{tabular}


Table 1 (continued).

\begin{tabular}{|c|c|c|c|c|c|c|c|c|c|c|c|c|c|c|c|}
\hline $\begin{array}{l}\text { Core, sample, } \\
\text { interval }(\mathrm{cm})\end{array}$ & $\begin{array}{l}\text { Depth } \\
\text { (mbsf) }\end{array}$ & $\begin{array}{l}\text { Age } \\
\text { (Ma) }\end{array}$ & $\begin{array}{c}\mathrm{SiO}_{2} \\
(\mathrm{wt} \%)\end{array}$ & $\begin{array}{l}\mathrm{TiO}_{2} \\
(\mathrm{wt} \%)\end{array}$ & $\begin{array}{l}\mathrm{Al}_{2} \mathrm{O}_{3} \\
(\mathrm{wt} \%)\end{array}$ & $\begin{array}{l}\mathrm{FeO} \\
(\mathrm{wt} \%)\end{array}$ & $\begin{array}{l}\mathrm{MnO} \\
(\mathrm{wt} \%)\end{array}$ & $\begin{array}{l}\mathrm{MgO} \\
\text { (wt\%) }\end{array}$ & $\begin{array}{l}\mathrm{CaO} \\
\text { (wt\%) }\end{array}$ & $\begin{array}{l}\mathrm{Na}_{2} \mathrm{O} \\
(\mathrm{wt} \%)\end{array}$ & $\begin{array}{c}\mathrm{K}_{2} \mathrm{O} \\
\text { (wt\%) }\end{array}$ & $\begin{array}{l}\mathrm{Cr}_{2} \mathrm{O}_{3} \\
(\mathrm{wt} \%)\end{array}$ & $\begin{array}{l}\mathrm{P}_{2} \mathrm{O}_{5} \\
(\mathrm{wt} \%)\end{array}$ & $\begin{array}{c}\text { Total } \\
\text { (wt\%) }\end{array}$ & \\
\hline $100 \mathrm{R}-2,27$ & 1215.77 & 52.00 & 48.196 & 0.544 & 17.835 & 17.826 & 0.000 & 2.366 & 0.547 & 0.849 & 1.325 & 0.039 & 0.113 & 89.640 & \\
\hline $100 \mathrm{R}-3,82$ & 1217.82 & 52.00 & 53.867 & 1.222 & 15.942 & 7.169 & 0.018 & 1.806 & 0.203 & 0.085 & 1.612 & 0.000 & 0.000 & 81.924 & \\
\hline $100 \mathrm{R}-3,82$ & 1217.82 & 52.00 & 60.518 & 0.391 & 17.936 & 9.544 & 0.033 & 2.363 & 0.196 & 0.563 & 0.952 & 0.000 & 0.041 & 92.537 & \\
\hline $100 \mathrm{R}-3,82$ & 1217.82 & 52.00 & 58.418 & 1.001 & 15.665 & 9.603 & 0.077 & 2.278 & 0.281 & 0.094 & 1.907 & 0.055 & 0.020 & 89.399 & \\
\hline $100 \mathrm{R}-3,82$ & 1217.82 & 52.00 & $\begin{array}{l}30.410 \\
57.667\end{array}$ & 0.671 & 18.193 & 8.381 & 0.011 & 2.158 & $\begin{array}{l}0.059 \\
0.059\end{array}$ & 0.181 & 1.420 & 0.028 & 0.007 & 88.776 & \\
\hline $100 \mathrm{R}-3,82$ & 1217.82 & 52.00 & 56.795 & 0.466 & 17.696 & 8.161 & 0.000 & 2.075 & 0.124 & 0.033 & 1.434 & 0.000 & 0.000 & 86.784 & \\
\hline 152-918A- & & & & & & & & & & & & & & & \\
\hline $2 \mathrm{H}-2,34$ & 3.64 & 0.04 & 49.492 & 3.056 & 13.551 & 14.281 & 0.235 & 5.740 & 10.017 & 2.636 & 0.400 & 0.007 & 0.288 & 99.703 & \\
\hline $2 \mathrm{H}-4,24$ & 6.54 & 0.08 & 47.203 & 2.470 & 14.736 & 9.645 & 0.195 & 7.795 & 12.616 & 2.176 & 0.849 & 0.030 & 0.347 & 98.062 & \\
\hline 152-919B- & & & & & & & & & & & & & & & \\
\hline $2 \mathrm{H}-1,43$ & 9.63 & 0.14 & 48.572 & 3.067 & 13.023 & 13.988 & 0.183 & 5.509 & 9.634 & 2.677 & 0.431 & 0.009 & 0.369 & 97.462 & \\
\hline $2 \mathrm{H}-1,43$ & 9.63 & 0.14 & 49.087 & 3.375 & 12.886 & 14.483 & 0.265 & 4.741 & 9.269 & 2.605 & 0.578 & 0.002 & 0.473 & 97.764 & \\
\hline $2 \mathrm{H}-1,106$ & 10.26 & 0.15 & 49.062 & 2.457 & 13.327 & 13.717 & 0.194 & 5 & $\begin{array}{l}9.2099 \\
10.299\end{array}$ & 2.529 & 0.349 & 0.063 & 0.272 & 98.172 & \\
\hline $2 \mathrm{H}-1,106$ & $\begin{array}{l}10.20 \\
10.26\end{array}$ & 0.15 & 49.066 & 2.653 & 12.914 & 14.344 & $\begin{array}{l}0.1943 \\
0.243\end{array}$ & 5.364 & 9.665 & 2.612 & 0.426 & 0.023 & 0.357 & 97.667 & \\
\hline $2 \mathrm{H}-1,106$ & 10.26 & 0.15 & 49.950 & 2.526 & 13.497 & 13.055 & 0.168 & 5.820 & 10.346 & 2.682 & 0.415 & 0.011 & 0.363 & 98.833 & \\
\hline $2 \mathrm{H}-1,106$ & 10.26 & 0.15 & 49.840 & 2.806 & 13.052 & 14.950 & 0.310 & 5.078 & 9.767 & 1.723 & 0.423 & 0.000 & 0.343 & 98.292 & \\
\hline $2 \mathrm{H}-1,106$ & 10.26 & 0.15 & 49.605 & 2.591 & 13.264 & 13.116 & 0.227 & 6.205 & 10.604 & 2.498 & 0.420 & $\begin{array}{l}0.000 \\
0.000\end{array}$ & $\begin{array}{l}0.343 \\
0.311\end{array}$ & $\begin{array}{l}98.292 \\
98.841\end{array}$ & \\
\hline $152-918 \mathrm{~A}-$ & & & & & & & & & & & & & & & \\
\hline $3 \mathrm{H}-3,142$ & 15.42 & 0.16 & 49.442 & 3.787 & 10.746 & 15.268 & 0.317 & 6.592 & 11.145 & 2.091 & 0.504 & 0.036 & 0.345 & 100.273 & \\
\hline $3 \mathrm{H}-3,142$ & 15.42 & 0.16 & 48.469 & 2.803 & 14.059 & 12.267 & 0.207 & 5.970 & 10.304 & 2.565 & 0.546 & 0.070 & 0.380 & 97.640 & \\
\hline $3 \mathrm{H}-3,142$ & 15.42 & 0.16 & 48.697 & 3.283 & 12.850 & 13.289 & 0.231 & 5.745 & 10.233 & 2.538 & 0.385 & 0.053 & 0.314 & 97.618 & \\
\hline $4 \mathrm{H}-2,48$ & 22.78 & 0.26 & 50.573 & 1.927 & 13.239 & 13.890 & 0.244 & 6.031 & 10.168 & 2.284 & 0.232 & 0.033 & 0.263 & 98.884 & \\
\hline $5 \mathrm{H}-3,103$ & 34.03 & 0.55 & 49.263 & $\begin{array}{l}1.927 \\
2.357\end{array}$ & 13.971 & 12.190 & 0.199 & $\begin{array}{l}0.632 \\
6.632\end{array}$ & $\begin{array}{l}10.100 \\
11.296\end{array}$ & 2.341 & 0.289 & 0.038 & $\begin{array}{l}0.210 \\
0.210\end{array}$ & $\begin{array}{l}90.004 \\
98.786\end{array}$ & \\
\hline $5 \mathrm{H}-3,103$ & 34.03 & 0.55 & 49.756 & 2.233 & 13.808 & 12.325 & 0.208 & 6.721 & 11.250 & 2.396 & 0.270 & 0.023 & 0.204 & 99.194 & \\
\hline $152-919 \mathrm{~A}-$ & & & & & & & & & & & & & & & \\
\hline $7 \mathrm{H}-6,135$ & 64.35 & 0.90 & 48.539 & 3.247 & 12.849 & 14.674 & 0.263 & 5.057 & 9.487 & 2.737 & 0.557 & 0.020 & 0.453 & 97.883 & \\
\hline $7 \mathrm{H}-6,135$ & 64.35 & 0.90 & 48.298 & 3.470 & 12.916 & 14.627 & 0.215 & 5.095 & 9.434 & 2.726 & 0.572 & 0.004 & 0.343 & 97.700 & \\
\hline $7 \mathrm{H}-6,135$ & 64.35 & 0.90 & 48.326 & 3.316 & 12.962 & 15.358 & 0.258 & 5.100 & 9.753 & 2.696 & 0.573 & 0.000 & 0.362 & 98.704 & \\
\hline $7 \mathrm{H}-6,135$ & 64.35 & 0.90 & 50.048 & 1.247 & 14.518 & 10.837 & 0.206 & 7.748 & 12.829 & 2.201 & 0.155 & 0.049 & 0.156 & 99.994 & \\
\hline $\begin{array}{c}\text { 152-918A- } \\
6 \mathrm{H}-1,122\end{array}$ & 41.02 & 1.00 & 67.722 & 0.042 & 18.638 & 1.902 & 0.024 & 0.870 & 0.383 & 8.244 & 2.087 & 0.000 & 0.000 & 99.912 & \\
\hline 152-919B- & & & & & & & & & & & & & & & \\
\hline $3 \mathrm{H}-3,85$ & 93.85 & 1.31 & 48.780 & 2.803 & 13.080 & 14.135 & 0.300 & 5.355 & 9.625 & 2.584 & 0.430 & 0.030 & 0.318 & 97.440 & \\
\hline $3 \mathrm{H}-3,85$ & 93.85 & 1.31 & 48.968 & 2.431 & 12.196 & 14.126 & 0.257 & 7.354 & 10.240 & 1.821 & 0.380 & 0.011 & 0.266 & 98.050 & \\
\hline $4 \mathrm{H}-3,115$ & 103.65 & 1.47 & 49.023 & 0.778 & 15.017 & 9.220 & 0.152 & 8.822 & 13.728 & 1.784 & 0.067 & 0.027 & 0.013 & 98.631 & \\
\hline $4 \mathrm{H}-3,115$ & 103.65 & 1.47 & 50.422 & 1.395 & 14.463 & 11.204 & 0.208 & 7.253 & 11.486 & 2.225 & 0.261 & 0.046 & 0.111 & 99.074 & \\
\hline $\begin{array}{r}\text { 152-918A- } \\
8 \mathrm{H}-2,34\end{array}$ & & & & & & & & & & & & & & & \\
\hline $\begin{array}{l}8 \mathrm{H}-2,34 \\
10 \mathrm{H}-3,2\end{array}$ & $\begin{array}{l}60.64 \\
80.82\end{array}$ & $\begin{array}{l}1.50 \\
1.60\end{array}$ & $\begin{array}{l}48.747 \\
49.721\end{array}$ & $\begin{array}{l}3.072 \\
2.767\end{array}$ & $\begin{array}{l}12.866 \\
13.260\end{array}$ & $\begin{array}{l}13.950 \\
13.355\end{array}$ & $\begin{array}{l}0.233 \\
0.201\end{array}$ & $\begin{array}{l}5.310 \\
5.977\end{array}$ & $\begin{array}{r}9.454 \\
10.102\end{array}$ & $\begin{array}{l}2.586 \\
2.593\end{array}$ & $\begin{array}{l}0.443 \\
0.404\end{array}$ & $\begin{array}{l}0.003 \\
0.045\end{array}$ & $\begin{array}{l}0.357 \\
0.195\end{array}$ & $\begin{array}{l}97.021 \\
98.620\end{array}$ & $\vec{\nabla}$ \\
\hline $10 \mathrm{H}-3,2$ & 80.82 & $\begin{array}{l}1.00 \\
1.60\end{array}$ & 49.088 & 2.379 & 13.385 & 12.953 & 0.227 & 5.917 & $\begin{array}{l}10.102 \\
10.732\end{array}$ & 2.493 & $\begin{array}{l}0.404 \\
0.279\end{array}$ & $\begin{array}{l}0.043 \\
0.031\end{array}$ & 0.253 & 97.737 & กิ \\
\hline 152-919B- & & & & & & & & & & & & & & & \\
\hline $5 \mathrm{H}-3,99$ & 112.99 & 1.60 & 49.672 & 3.363 & 13.158 & 15.085 & 0.291 & 5.029 & 9.268 & 2.491 & 0.652 & 0.000 & 0.337 & 99.346 & z \\
\hline $5 \mathrm{H}-3,99$ & 112.99 & 1.60 & 49.721 & 3.495 & 13.626 & 15.618 & 0.209 & 4.649 & 8.997 & 1.999 & 0.607 & 0.018 & 0.389 & 99.328 & $\theta$ \\
\hline $5 \mathrm{H}-3,99$ & 112.99 & 1.60 & 48.372 & 3.507 & 12.897 & 15.007 & 0.295 & 5.072 & 9.487 & 2.729 & 0.529 & 0.000 & 0.401 & 98.296 & $\pi$ \\
\hline $5 \mathrm{H}-3,99$ & 112.99 & 1.60 & 48.555 & 3.307 & 12.879 & 14.364 & 0.226 & 5.150 & 9.340 & 2.702 & 0.563 & 0.036 & 0.511 & 97.633 & d \\
\hline $5 \mathrm{H}-3,99$ & 112.99 & 1.60 & 49.110 & 3.327 & 12.981 & 14.059 & 0.308 & 5.354 & 10.127 & 2.444 & 0.646 & 0.031 & 0.440 & 98.827 & $\mathbb{\pi}$ \\
\hline $5 \mathrm{H}-3,99$ & 112.99 & 1.60 & 49.291 & 3.318 & 13.607 & 14.416 & 0.183 & 4.290 & 9.109 & 2.910 & 0.587 & 0.024 & 0.460 & 98.195 & (1) \\
\hline $6 \mathrm{H}-1,43$ & 118.93 & 1.69 & 48.572 & 3.067 & 13.023 & 13.988 & 0.183 & 5.509 & 9.634 & 2.677 & 0.431 & 0.009 & 0.369 & 97.462 & 分 \\
\hline 152-919B- & & & & & & & & & & & & & & & 元 \\
\hline $6 \mathrm{H}-1,43$ & 118.93 & 1.69 & 49.087 & 3.375 & 12.886 & 14.483 & 0.265 & 4.741 & 9.269 & 2.605 & 0.578 & 0.002 & 0.473 & 97.764 & (T) \\
\hline $6 \mathrm{H}-6,87$ & 126.87 & 1.77 & 48.893 & 2.927 & 13.155 & 14.142 & 0.257 & 5.457 & 9.635 & 2.749 & 0.397 & 0.000 & 0.266 & 97.878 & $E$ \\
\hline $6 \mathrm{H}-6,87$ & 126.87 & 1.77 & 48.994 & 2.767 & 12.959 & 14.955 & 0.265 & 5.246 & 9.190 & 2.704 & 0.414 & 0.000 & 0.344 & 97.838 & 茴 \\
\hline $6 \mathrm{H}-6,87$ & 126.87 & 1.77 & 48.842 & 2.579 & 13.025 & 14.007 & 0.257 & 5.425 & 9.849 & 2.730 & 0.391 & 0.033 & 0.266 & 97.404 & 3 \\
\hline $6 \mathrm{H}-6,87$ & 126.87 & 1.77 & 48.787 & 2.561 & 13.042 & 13.171 & 0.212 & 6.390 & 11.736 & 2.599 & 0.183 & 0.009 & 0.252 & 98.942 & $\stackrel{\pi}{Z}$ \\
\hline $6 \mathrm{H}-6,87$ & 126.87 & 1.77 & 50.404 & 2.603 & 13.253 & 13.034 & 0.266 & 5.368 & 9.345 & 2.802 & 0.545 & 0.000 & 0.351 & 97.971 & 莺 \\
\hline $\begin{array}{c}\text { 152-918A- } \\
11 \mathrm{H}-2,31\end{array}$ & 89.11 & 1.80 & 49.314 & 2.076 & 13.948 & 11.525 & 0.227 & 6.795 & 11.089 & 2.490 & 0.303 & 0.000 & 0.182 & 97.949 & 胥 \\
\hline 152-919B- & & & & & & & & & & & & & & & 3 \\
\hline $7 \mathrm{H}-1,37$ & 128.37 & 1.80 & 48.317 & 3.397 & 12.964 & 15.235 & 0.305 & 5.061 & 9.704 & 2.676 & 0.537 & 0.022 & 0.355 & 98.573 & 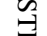 \\
\hline $7 \mathrm{H}-1,37$ & 128.37 & 1.80 & 70.894 & 0.226 & 7.827 & 6.389 & 0.281 & 0.019 & 0.275 & 4.027 & 2.811 & 0.047 & 0.007 & 92.803 & $\vec{x}$ \\
\hline
\end{tabular}


Table 1 (continued).

\begin{tabular}{|c|c|c|c|c|c|c|c|c|c|c|c|c|c|c|}
\hline $\begin{array}{l}\text { Core, sample, } \\
\text { interval }(\mathrm{cm})\end{array}$ & $\begin{array}{l}\text { Depth } \\
\text { (mbsf) }\end{array}$ & $\begin{array}{l}\text { Age } \\
\text { (Ma) }\end{array}$ & $\begin{array}{c}\mathrm{SiO}_{2} \\
(\mathrm{wt} \%)\end{array}$ & $\begin{array}{l}\mathrm{TiO}_{2} \\
(\mathrm{wt} \%)\end{array}$ & $\begin{array}{l}\mathrm{Al}_{2} \mathrm{O}_{3} \\
(\mathrm{wt} \%)\end{array}$ & $\begin{array}{l}\mathrm{FeO} \\
(\mathrm{w} \%)\end{array}$ & $\begin{array}{l}\mathrm{MnO} \\
(\mathrm{wt} \%)\end{array}$ & $\begin{array}{l}\mathrm{MgO} \\
(\mathrm{w} \%)\end{array}$ & $\begin{array}{l}\mathrm{CaO} \\
(\mathrm{wt} \%)\end{array}$ & $\begin{array}{l}\mathrm{Na}_{2} \mathrm{O} \\
(\mathrm{wt} \%)\end{array}$ & $\begin{array}{l}\mathrm{K}_{2} \mathrm{O} \\
(\mathrm{wt} \%)\end{array}$ & $\begin{array}{l}\mathrm{Cr}_{2} \mathrm{O}_{3} \\
(\mathrm{wt} \%)\end{array}$ & $\begin{array}{l}\mathrm{P}_{2} \mathrm{O}_{5} \\
(\mathrm{wt} \%)\end{array}$ & $\begin{array}{c}\text { Total } \\
\text { (wt\%) }\end{array}$ \\
\hline \multicolumn{15}{|l|}{ 152-918A- } \\
\hline $13 \mathrm{H}-6,35$ & 114.15 & 2.25 & 48.382 & 2.107 & 13.778 & 11.900 & 0.221 & 6.936 & 11.499 & 2.474 & 0.202 & 0.044 & 0.110 & 97.653 \\
\hline $13 \mathrm{H}-6,35$ & 114.15 & 2.25 & 48.286 & 2.061 & 13.820 & 12.165 & 0.173 & 6.917 & 11.719 & 2.482 & 0.176 & 0.008 & 0.201 & 98.008 \\
\hline $13 \mathrm{H}-6,35$ & 114.15 & 2.25 & 48.140 & 1.878 & 13.570 & 11.965 & 0.238 & 6.942 & 11.406 & 2.376 & 0.203 & 0.013 & 0.175 & 96.906 \\
\hline $13 \mathrm{H}-6,35$ & 114.15 & 2.25 & 48.226 & 2.054 & 13.551 & 12.423 & 0.242 & 6.796 & 11.366 & 2.441 & 0.243 & 0.022 & 0.253 & 97.617 \\
\hline $14 \mathrm{H}-3,0$ & 118.8 & 2.30 & 49.120 & 2.408 & 13.446 & 13.060 & 0.158 & 6.230 & 10.441 & 2.524 & 0.364 & 0.018 & 0.143 & 97.912 \\
\hline $14 \mathrm{H}-3,0$ & 118.8 & 2.30 & 48.877 & 2.493 & 13.265 & 13.375 & 0.205 & 5.898 & 10.557 & 2.528 & 0.291 & 0.000 & 0.227 & 97.716 \\
\hline $14 \mathrm{H}-3,0$ & 118.8 & 2.30 & 49.230 & 1.805 & 14.220 & 11.184 & 0.158 & 7.280 & 11.934 & 2.306 & 0.283 & 0.050 & 0.124 & 98.574 \\
\hline $14 \mathrm{H}-3,0$ & 118.8 & 2.30 & 47.020 & 3.086 & 15.559 & 11.899 & 0.162 & 5.491 & 8.862 & 3.679 & 1.148 & 0.051 & 0.636 & 97.593 \\
\hline $18 \mathrm{H}-\mathrm{CC}, 14$ & 161.8 & 2.50 & 71.092 & 0.028 & 12.582 & 1.486 & 0.087 & 0.034 & 0.646 & 2.335 & 5.409 & 0.000 & 0.000 & 93.699 \\
\hline $18 \mathrm{H}-\mathrm{CC}, 14$ & 161.8 & 2.50 & 48.435 & 3.824 & 13.033 & 13.374 & 0.296 & 4.564 & 8.965 & 3.075 & 1.476 & 0.000 & 1.062 & 98.104 \\
\hline $20 \mathrm{H}-3,9$ & 174.39 & 2.52 & 49.337 & 1.375 & 15.247 & 10.317 & 0.188 & 7.985 & 12.051 & 2.118 & 0.408 & 0.056 & 0.098 & 99.180 \\
\hline $21 \mathrm{H}-2,109$ & 184.09 & 2.55 & 49.898 & 3.692 & 12.713 & 14.842 & 0.267 & 4.809 & 8.781 & 2.450 & 0.558 & 0.043 & 0.377 & 98.430 \\
\hline $21 \mathrm{H}-2,109$ & 184.09 & 2.55 & 52.061 & 3.565 & 12.550 & 13.914 & 0.331 & 3.625 & 7.708 & 2.993 & 0.793 & 0.048 & 0.508 & 98.096 \\
\hline $21 \mathrm{H}-2,109$ & 184.09 & 2.55 & 50.841 & 3.217 & 13.332 & 13.614 & 0.204 & 4.876 & 8.954 & 2.783 & 0.648 & 0.014 & 0.397 & 98.880 \\
\hline $21 \mathrm{H}-2,109$ & 184.09 & 2.55 & 52.274 & 3.492 & 12.661 & 14.916 & 0.330 & 3.812 & 8.247 & 2.731 & 0.721 & 0.000 & 0.540 & 99.724 \\
\hline $23 X-5,14$ & 205.94 & 2.60 & 49.765 & 3.247 & 12.904 & 14.830 & 0.234 & 4.990 & 9.328 & 2.783 & 0.512 & 0.000 & 0.240 & 98.833 \\
\hline $23 X-5,14$ & 205.94 & 2.60 & 48.868 & 2.505 & 14.024 & 12.437 & 0.193 & 6.716 & 10.912 & 2.480 & 0.276 & 0.011 & 0.208 & 98.630 \\
\hline $23 \mathrm{X}-5,67$ & 206.47 & 2.63 & 48.940 & 2.242 & 13.641 & 13.599 & 0.204 & 6.680 & 10.848 & 2.305 & 0.248 & & 0.221 & 98.983 \\
\hline $23 \mathrm{X}-5,67$ & 206.47 & 2.63 & 49.034 & 2.355 & 13.784 & 13.225 & 0.255 & 6.654 & 10.756 & 2.365 & 0.274 & 0.028 & 0.221 & 98.951 \\
\hline $23 X-5,67$ & 206.47 & 2.63 & 49.096 & 2.408 & 13.904 & 13.123 & 0.160 & 6.932 & 10.776 & 2.352 & & 0.051 & 0.279 & 99.308 \\
\hline $23 X-5,67$ & 206.47 & 2.63 & 49.179 & 2.367 & 13.708 & 13.727 & 0.200 & 6.426 & 10.625 & 2.388 & 0.221 & 0.038 & 0.208 & 99.087 \\
\hline $23 X-5,122$ & 207.02 & 2.65 & 49.165 & 3.445 & 13.141 & 14.605 & 0.258 & 5.862 & 10.089 & 2.290 & 0.396 & 0.002 & 0.434 & 99.687 \\
\hline $23 X-5,122$ & 207.02 & 2.65 & 47.875 & 1.960 & 15.501 & 12.652 & 0.224 & 7.835 & 11.089 & 2.343 & 0.246 & 0.007 & 0.156 & 99.888 \\
\hline $23 X-5,122$ & 207.02 & 2.65 & 51.239 & 2.767 & 13.165 & 13.657 & 0.261 & 4.447 & 8.710 & 2.746 & 0.703 & 0.000 & 0.489 & 98.184 \\
\hline $23 \mathrm{X}-5,122$ & 212.98 & 2.70 & 48.916 & 2.051 & 13.910 & 11.975 & 0.202 & 6.966 & 11.669 & 2.329 & 0.231 & 0.000 & 0.189 & 98.438 \\
\hline \multicolumn{15}{|l|}{$152-918 \mathrm{~A}-$} \\
\hline $24 \mathrm{X}-3,128$ & 212.98 & 2.70 & 49.101 & 2.713 & 13.379 & 13.280 & 0.198 & 6.122 & 10.416 & 2.475 & 0.292 & 0.035 & 0.299 & 98.310 \\
\hline $24 \mathrm{X}-3,128$ & 212.98 & 2.70 & 48.256 & 3.178 & 13.138 & 14.903 & 0.217 & 5.631 & 10.443 & 2.672 & 0.346 & 0.000 & 0.388 & 99.172 \\
\hline $25 \mathrm{X}-4,109$ & 223.19 & 2.75 & 50.024 & 1.245 & 13.548 & 12.358 & 0.207 & 6.792 & 11.360 & 2.121 & 0.137 & 0.007 & 0.131 & 97.930 \\
\hline $25 \mathrm{X}-4,109$ & 223.19 & 2.75 & 43.104 & 1.339 & 12.917 & 14.923 & 0.219 & 11.577 & 10.823 & 1.234 & 0.968 & 0.029 & 0.000 & 97.133 \\
\hline $25 \mathrm{X}-7,4$ & 226.64 & 2.80 & 49.337 & 3.221 & 13.320 & 14.065 & 0.197 & 5.268 & 9.466 & 2.695 & 0.563 & 0.006 & 0.480 & 98.618 \\
\hline $25 \mathrm{X}-7,4$ & 226.64 & 2.80 & 50.031 & 3.041 & 13.399 & 13.956 & 0.239 & 5.164 & 9.313 & 2.728 & 0.563 & 0.000 & 0.390 & 98.824 \\
\hline $25 \mathrm{X}-7,4$ & 226.64 & 2.80 & 50.434 & 3.162 & 13.427 & 13.572 & 0.195 & 5.267 & 9.337 & 2.732 & 0.559 & 0.050 & 0.436 & 99.171 \\
\hline $25 \mathrm{X}-7,4$ & 226.64 & 2.80 & 51.044 & 3.018 & 13.264 & 13.908 & 0.224 & 4.828 & 8.656 & 2.764 & 0.621 & 0.020 & 0.495 & 98.842 \\
\hline $28 X-2,67$ & 246.27 & 2.90 & 55.398 & 0.122 & 27.815 & 1.412 & 0.000 & 0.292 & 10.567 & 5.194 & 0.348 & 0.000 & 0.020 & 101.168 \\
\hline $33 X-2,59$ & 290.49 & 3.20 & 48.098 & 3.170 & 12.816 & 15.851 & 0.252 & 5.032 & 9.495 & 2.700 & 0.532 & 0.000 & 0.383 & 98.329 \\
\hline $33 \mathrm{X}-2,59$ & 290.49 & 3.20 & 49.446 & 1.751 & 13.748 & 12.835 & 0.221 & 6.501 & 11.329 & 2.423 & 0.345 & 0.015 & 0.157 & 98.771 \\
\hline $33 X-2,59$ & 290.49 & 3.20 & 49.201 & 2.395 & 13.361 & 12.991 & 0.189 & 5.886 & 10.345 & 2.665 & 0.437 & 0.001 & 0.313 & 97.784 \\
\hline $33 \mathrm{X}-2,59$ & 290.49 & 3.20 & 49.116 & 2.319 & 13.813 & 12.409 & 0.226 & 6.596 & 11.496 & 2.531 & 0.331 & 0.025 & 0.208 & 99.070 \\
\hline \multicolumn{15}{|l|}{ 152-918D- } \\
\hline 36R-1, 17 & 619.07 & 10.50 & 45.012 & 0.402 & 12.451 & 14.356 & 0.223 & 11.153 & 12.155 & 1.039 & 0.738 & 0.061 & 0.000 & 97.590 \\
\hline $39 \mathrm{R}-4,14$ & 652.54 & 12.10 & 73.278 & 0.065 & 11.965 & 1.253 & 0.101 & 0.169 & 1.151 & 2.923 & 2.900 & 0.024 & 0.000 & 93.829 \\
\hline $40 \mathrm{R}-2,36$ & 659.36 & 12.50 & 71.006 & 0.303 & 11.791 & 3.400 & 0.146 & 0.068 & 1.852 & 3.603 & 1.830 & 0.000 & 0.014 & 94.013 \\
\hline $40 \mathrm{R}-2,36$ & 659.36 & 12.50 & 70.738 & 0.194 & 11.712 & 3.560 & 0.072 & 0.093 & 1.905 & 3.597 & 1.791 & 0.026 & 0.034 & 93.722 \\
\hline $40 \mathrm{R}-3,114$ & 661.64 & 12.70 & 72.999 & 0.155 & 11.505 & 1.501 & 0.061 & 0.076 & 0.910 & 3.218 & 3.008 & 0.016 & 0.028 & 93.477 \\
\hline $40 \mathrm{R}-3,114$ & 661.64 & 12.70 & 70.758 & 0.263 & 11.909 & 3.614 & 0.137 & 0.116 & 1.787 & 3.343 & 1.980 & 0.010 & 0.048 & 93.965 \\
\hline $40 \mathrm{R}-3,114$ & 661.64 & 12.70 & 70.901 & 0.279 & 11.749 & 3.377 & 0.101 & 0.145 & 1.750 & 3.429 & 1.883 & 0.009 & 0.041 & 93.664 \\
\hline 40R-3, 114 & 661.64 & 12.70 & 70.502 & 0.279 & 11.898 & 3.384 & 0.117 & 0.148 & 1.754 & 3.154 & 1.964 & 0.000 & 0.014 & 93.214 \\
\hline $51 \mathrm{R}-6,67$ & 771.37 & 17.40 & 59.942 & 0.270 & 10.906 & 1.044 & 0.034 & 0.921 & 1.030 & 3.120 & 2.272 & 0.017 & 0.000 & 79.556 \\
\hline \multicolumn{15}{|l|}{ 152-914B- } \\
\hline 16R-1, 90 & 226.7 & 32.10 & 48.809 & 0.467 & 8.705 & 11.964 & 0.113 & 14.591 & 11.177 & 1.386 & 0.176 & 0.145 & 0.000 & 97.533 \\
\hline $16 \mathrm{R}-1,90$ & 226.7 & 32.10 & 44.187 & 1.570 & 12.306 & 13.485 & 0.179 & 11.881 & 11.397 & 1.543 & 0.926 & 0.127 & 0.046 & 97.647 \\
\hline $17 \mathrm{R}-1,146$ & 236.96 & 32.90 & 43.119 & 2.111 & 10.689 & 14.703 & 0.376 & 12.201 & 11.508 & 1.692 & 1.589 & 0.000 & 0.000 & 97.988 \\
\hline \multicolumn{15}{|l|}{ 152-918D- } \\
\hline $88 \mathrm{R}-1,42$ & 1118.22 & 39.70 & 53.759 & 0.336 & 9.671 & 11.777 & 0.111 & 6.840 & 0.194 & 0.558 & 2.804 & 0.015 & 0.107 & 86.172 \\
\hline $89 \mathrm{R}-4,49$ & 1132.39 & 44.04 & 54.750 & 0.967 & 12.368 & 10.643 & 0.007 & 3.493 & 0.490 & 0.383 & 3.987 & 0.039 & 0.321 & 87.448 \\
\hline $89 \mathrm{R}-4,49$ & 1132.39 & 44.04 & 59.404 & 0.768 & 7.335 & 12.798 & 0.000 & 2.782 & 0.229 & 0.229 & 4.646 & 0.013 & 0.113 & 88.317 \\
\hline $89 \mathrm{R}-4,49$ & 1132.39 & 44.04 & 59.800 & 0.760 & 7.649 & 12.397 & 0.020 & 2.751 & 0.230 & 0.242 & 4.432 & 0.000 & 0.167 & 88.448 \\
\hline $89 \mathrm{R}-4,49$ & 1132.39 & 44.04 & 70.839 & 0.473 & 3.506 & 5.275 & 0.007 & 1.049 & 0.296 & 0.286 & 1.523 & 0.000 & 0.110 & 83.364 \\
\hline $89 \mathrm{R}-4,49$ & 1132.39 & 44.04 & 59.861 & 1.241 & 8.154 & 7.392 & 0.070 & 2.064 & 0.292 & 0.331 & 2.564 & 0.031 & 0.312 & 82.312 \\
\hline $91 \mathrm{R}-2,10$ & 1148.1 & 47.90 & 53.499 & 3.886 & 14.083 & 7.483 & 0.048 & 2.712 & 0.634 & 0.479 & 1.153 & 0.010 & 0.067 & 84.054 \\
\hline $93 \mathrm{R}-2,64$ & 1167.94 & 48.30 & 61.268 & 0.955 & 4.410 & 10.304 & 0.000 & 1.747 & 0.123 & 0.636 & 3.060 & 0.000 & 0.364 & 82.867 \\
\hline 93R-2, 64 & 1167.94 & 48.30 & 61.358 & 1.623 & 10.384 & 7.021 & 0.002 & 3.301 & 0.158 & 1.205 & 1.795 & 0.076 & 0.238 & 87.161 \\
\hline
\end{tabular}


Table 1 (continued).

\begin{tabular}{|c|c|c|c|c|c|c|c|c|c|c|c|c|c|c|}
\hline $\begin{array}{l}\text { Core, sample, } \\
\text { interval }(\mathrm{cm})\end{array}$ & $\begin{array}{l}\text { Depth } \\
\text { (mbsf) }\end{array}$ & $\begin{array}{l}\text { Age } \\
(\mathrm{Ma})\end{array}$ & $\begin{array}{c}\mathrm{SiO}_{2} \\
(\mathrm{wt} \%)\end{array}$ & $\begin{array}{c}\mathrm{TiO}_{2} \\
(\mathrm{wt} \%)\end{array}$ & $\begin{array}{l}\mathrm{Al}_{2} \mathrm{O}_{3} \\
\text { (wt\%) }\end{array}$ & $\begin{array}{c}\mathrm{FeO} \\
(\mathrm{wt} \%)\end{array}$ & $\begin{array}{l}\mathrm{MnO} \\
(\mathrm{wt} \%)\end{array}$ & $\begin{array}{l}\mathrm{MgO} \\
\text { (wt\%) }\end{array}$ & $\begin{array}{l}\mathrm{CaO} \\
(\mathrm{wt} \%)\end{array}$ & $\begin{array}{l}\mathrm{Na}_{2} \mathrm{O} \\
(\mathrm{wt} \%)\end{array}$ & $\begin{array}{c}\mathrm{K}_{2} \mathrm{O} \\
(\mathrm{wt} \%)\end{array}$ & $\begin{array}{l}\mathrm{Cr}_{2} \mathrm{O}_{3} \\
(\mathrm{wt} \%)\end{array}$ & $\begin{array}{l}\mathrm{P}_{2} \mathrm{O}_{5} \\
(\mathrm{wt} \%)\end{array}$ & $\begin{array}{l}\text { Total } \\
\text { (wt\%) }\end{array}$ \\
\hline \multicolumn{15}{|l|}{ 152-918D- } \\
\hline $95 \mathrm{R}-3,94$ & 1184.34 & 50.60 & 49.675 & 0.172 & 9.046 & 9.659 & 0.221 & 15.956 & 11.153 & 1.216 & 0.163 & 0.325 & 0.059 & 97.645 \\
\hline $96 \mathrm{R}-1,29$ & 1185.39 & 51.00 & 43.453 & 1.231 & 11.313 & 17.942 & 0.272 & 9.517 & 11.961 & 1.234 & 1.000 & 0.000 & 0.000 & 97.923 \\
\hline 96R-1, 29 & 1185.39 & 51.00 & $\begin{array}{l}43.453 \\
58.721\end{array}$ & 0.539 & 3.985 & 16.227 & 0.052 & 2.596 & 0.087 & $\begin{array}{l}1.070 \\
0.070\end{array}$ & $\begin{array}{l}1.607 \\
4.667\end{array}$ & 0.015 & 0.066 & 87.025 \\
\hline 96R-3, 120 & 1189.3 & 51.40 & 54.901 & 2.305 & 13.682 & 9.528 & 0.018 & 3.387 & 0.377 & 0.139 & 1.171 & 0.007 & 0.108 & 85.623 \\
\hline $100 \mathrm{R}-2,27$ & 1215.77 & 52.00 & 49.034 & 0.234 & 14.082 & 17.583 & 0.052 & 2.843 & 0.786 & 0.454 & 2.493 & 0.028 & 0.040 & 87.629 \\
\hline $100 \mathrm{R}-2,27$ & 1215.77 & 52.00 & 48.196 & 0.544 & 17.835 & 17.826 & 0.000 & 2.366 & 0.547 & 0.849 & 1.325 & 0.039 & 0.113 & 89.640 \\
\hline $100 \mathrm{R}-3,82$ & 1217.82 & 52.00 & 53.867 & 1.222 & 15.942 & 7.169 & 0.018 & 1.806 & 0.203 & 0.085 & 1.612 & 0.000 & 0.000 & 81.924 \\
\hline $100 \mathrm{R}-3,82$ & 1217.82 & 52.00 & 60.518 & 0.391 & 17.936 & 9.544 & 0.033 & 2.363 & 0.196 & 0.563 & 0.952 & 0.000 & 0.041 & 92.537 \\
\hline $100 \mathrm{R}-3,82$ & 1217.82 & 52.00 & 58.418 & 1.001 & 15.665 & 9.603 & 0.077 & 2.278 & 0.281 & 0.094 & 1.907 & 0.055 & 0.020 & 89.399 \\
\hline $100 \mathrm{R}-3,82$ & 1217.82 & 52.00 & 57.667 & 0.671 & 18.193 & 8.381 & 0.011 & 2.158 & 0.059 & 0.181 & 1.420 & 0.028 & 0.007 & 88.776 \\
\hline $100 \mathrm{R}-3,82$ & 1217.82 & 52.00 & 56.795 & 0.466 & 17.696 & 8.161 & 0.000 & 2.075 & 0.124 & 0.033 & 1.434 & 0.000 & 0.000 & 86.784 \\
\hline
\end{tabular}

Table 2. Trace and rare earth element concentrations for individual glass grains measured by ion probe.

\begin{tabular}{|c|c|c|c|c|c|c|c|c|c|c|c|c|c|c|c|c|c|c|c|c|c|c|}
\hline $\begin{array}{l}\text { Core, section } \\
\text { interval }(\mathrm{cm})\end{array}$ & $\begin{array}{l}\text { Depth } \\
\text { (mbsf) }\end{array}$ & $\begin{array}{l}\text { Age } \\
\text { (Ma) }\end{array}$ & $\begin{array}{c}\mathrm{Zr} \\
(\mathrm{ppm})\end{array}$ & $\begin{array}{c}\mathrm{Rb} \\
(\mathrm{ppm})\end{array}$ & $\begin{array}{c}\mathrm{Sr} \\
(\mathrm{ppm})\end{array}$ & $\begin{array}{c}\mathrm{Y} \\
(\mathrm{ppm})\end{array}$ & $\begin{array}{c}\mathrm{Nb} \\
(\mathrm{ppm})\end{array}$ & $\begin{array}{c}\mathrm{Ba} \\
(\mathrm{ppm})\end{array}$ & $\begin{array}{c}\mathrm{La} \\
(\mathrm{ppm})\end{array}$ & $\begin{array}{c}\mathrm{Ce} \\
(\mathrm{ppm})\end{array}$ & $\begin{array}{c}\mathrm{Pr} \\
(\mathrm{ppm})\end{array}$ & $\begin{array}{c}\mathrm{Nd} \\
(\mathrm{ppm})\end{array}$ & $\begin{array}{l}\mathrm{Sm} \\
(\mathrm{ppm})\end{array}$ & $\begin{array}{c}\mathrm{Eu} \\
(\mathrm{ppm})\end{array}$ & $\begin{array}{c}\mathrm{Gd} \\
(\mathrm{ppm})\end{array}$ & $\begin{array}{c}\mathrm{Tb} \\
(\mathrm{ppm})\end{array}$ & $\begin{array}{c}\text { Dy } \\
(\mathrm{ppm})\end{array}$ & $\begin{array}{c}\text { Ho } \\
(\mathrm{ppm})\end{array}$ & $\begin{array}{c}\mathrm{Er} \\
(\mathrm{ppm})\end{array}$ & $\begin{array}{c}\mathrm{Tm} \\
(\mathrm{ppm})\end{array}$ & $\begin{array}{c}\mathrm{Lu} \\
(\mathrm{ppm})\end{array}$ & $\begin{array}{c}\mathrm{U} \\
(\mathrm{ppm})\end{array}$ \\
\hline \multicolumn{23}{|l|}{$152-918 \mathrm{~A}-$} \\
\hline $2 \mathrm{H}-2,34$ & 3.64 & 0.04 & 252 & 7.03 & 185 & 39.6 & 21.8 & 101.0 & 19.2 & 47.9 & 6.93 & 31.0 & 3.62 & 2.33 & 12.00 & 1.810 & 10.80 & 2.27 & 6.51 & 0.907 & 0.892 & 0.338 \\
\hline $2 \mathrm{H}-2,34$ & 3.64 & 0.04 & 155 & 13.90 & 430 & 18.3 & 35.8 & 296.0 & 29.4 & 62.1 & 8.01 & 31.9 & 6.13 & 1.56 & 7.32 & 0.962 & $\begin{array}{r}4.69 \\
\end{array}$ & 0.94 & 3.39 & 0.493 & 0.453 & 0.447 \\
\hline $2 \mathrm{H}-2,34$ & 3.64 & 0.04 & 310 & 40.40 & 475 & 33.5 & 83.2 & 549.0 & 51.2 & 113 & $\begin{array}{l}0.01 \\
13.7\end{array}$ & 57.6 & 9.48 & 2.61 & 10.40 & 1.450 & 7.87 & 1.57 & 4.49 & 0.688 & 0.731 & 0.945 \\
\hline $3 \mathrm{H}-2,15$ & 12.95 & 0.13 & 199 & 6.21 & 186 & 34.2 & 17.9 & 84.7 & 16.3 & 39.9 & 5.65 & 25.8 & 5.87 & 2.12 & 10.90 & 1.470 & 8.99 & 1.64 & 5.39 & 0.855 & 0.815 & 0.294 \\
\hline $3 \mathrm{H}-3,112$ & 15.42 & 0.16 & 200 & $\begin{array}{l}0.21 \\
5.19\end{array}$ & $\begin{array}{l}100 \\
218\end{array}$ & 30.3 & 19.3 & $\begin{array}{l}806.0 \\
106.0\end{array}$ & $\begin{array}{l}10.5 \\
18.9\end{array}$ & 44.5 & $\begin{array}{l}5.21 \\
6.21\end{array}$ & 27.8 & 7.68 & 2.35 & 9.43 & 1.470 & 8.90 & $\begin{array}{l}1.04 \\
1.67\end{array}$ & 4.87 & 0.678 & $\begin{array}{l}0.013 \\
0.689\end{array}$ & 0.298 \\
\hline $3 \mathrm{H}-3,112$ & 15.42 & 0.16 & 125 & 2.75 & 248 & 20.0 & 11.7 & 73.1 & 10.8 & 25.4 & $\begin{array}{l}0.21 \\
3.8\end{array}$ & 16. & $\begin{array}{l}.000 \\
3.92\end{array}$ & 1.66 & $\begin{array}{l}5.45 \\
5.54\end{array}$ & $\begin{array}{l}1.470 \\
0.962\end{array}$ & $\begin{array}{l}0.90 \\
5.86\end{array}$ & 1.14 & $\begin{array}{l}\text { l.ol } \\
3.26\end{array}$ & $\begin{array}{l}0.078 \\
0.449\end{array}$ & $\begin{array}{l}0.089 \\
0.462\end{array}$ & 0.154 \\
\hline $4 \mathrm{H}-2,48$ & 22.78 & 0.26 & 132 & 1.53 & 106 & 32.6 & 11.9 & 57.9 & 11.2 & 26.7 & 3.87 & 18.4 & 4.73 & 1.60 & 9.03 & 1.370 & 8.42 & 1.74 & 5.80 & 0.741 & 0.881 & 0.176 \\
\hline $4 \mathrm{H}$ & 22.78 & 0.26 & 135 & 3.64 & 109 & 33.2 & 12.3 & 58.9 & 9. & 24.1 & 3.74 & $\begin{array}{l}10.4 \\
16.7\end{array}$ & 4.37 & $\begin{array}{l}1.00 \\
1.63\end{array}$ & 5.39 & $\begin{array}{l}1.070 \\
1.070\end{array}$ & $\begin{array}{l}8.42 \\
6.49\end{array}$ & $\begin{array}{l}1 . / 4 \\
1.44\end{array}$ & $\begin{array}{l}5.80 \\
4.75\end{array}$ & $\begin{array}{l}0.141 \\
0.739\end{array}$ & $\begin{array}{l}0.881 \\
0.760\end{array}$ & $\begin{array}{l}0.176 \\
0.091\end{array}$ \\
\hline $5 \mathrm{H}-3,103$ & 34.03 & 0.55 & 141 & 2.84 & 161 & 27.7 & 11.6 & 57.5 & 11.6 & 29.1 & 3.89 & $\begin{array}{l}10.1 \\
19.3\end{array}$ & 4.95 & $\begin{array}{l}1.03 \\
1.60\end{array}$ & 7.87 & 1.210 & $\begin{array}{l}6.49 \\
6.66\end{array}$ & 1.34 & $\begin{array}{l}4.17 \\
4.17\end{array}$ & 0.608 & 0.685 & 0.102 \\
\hline $5 \mathrm{H}-3,103$ & 34.03 & 0.55 & 158 & 4.51 & 180 & 29.1 & 13.5 & 68.6 & 12.1 & 29.6 & 4.4 & 20.8 & 5.5 & 1.60 & 5.62 & 1.180 & 7.47 & 1.37 & 4.26 & 0.554 & 0.626 & 0.114 \\
\hline $8 \mathrm{H}-2,34$ & 60.64 & 1.5 & 251 & 6.40 & 203 & 41.3 & 25.2 & 96.9 & 18.6 & 45.8 & 5.97 & 30.0 & 6.83 & 2.72 & 9.95 & 1.690 & 10.00 & 1.78 & 5.14 & 0.675 & 0.556 & 0.280 \\
\hline $8 \mathrm{H}-2,34$ & 60.64 & 1.5 & 266 & 6.46 & 194 & 43.1 & 27.0 & 101.0 & 19.6 & 47.2 & 6.25 & 30.9 & 8.31 & 2.34 & 9.95 & 1.630 & 8.90 & 1.86 & 5.37 & 0.856 & 0.688 & 0.362 \\
\hline $8 \mathrm{H}-2,34$ & 60.64 & 1.5 & 141 & 2.56 & 162 & 29.8 & 12.8 & 52.0 & 9.8 & 25.7 & 3.76 & 16.6 & 4.13 & 1.68 & 5.85 & 0.980 & 6.06 & 1.27 & 3.85 & 0.483 & 0.574 & 0.025 \\
\hline $10 \mathrm{H}-3,2$ & 80.82 & 1.6 & 153 & 3.65 & 166 & 31.7 & 11.9 & 60.0 & 12.2 & 30.6 & 4.64 & 22.1 & 5.56 & 1.98 & 9.83 & 1.380 & 7.80 & 1.66 & 4.81 & 0.745 & 0.584 & 0.776 \\
\hline $10 \mathrm{H}-3,2$ & 80.82 & 1.6 & 218 & 4.91 & 177 & 38.2 & 17.1 & 85.2 & 16.6 & 40.5 & 5.69 & 24 & 6.99 & 2.48 & 12.90 & 1.880 & 11.10 & 2. & 6.26 & 0.819 & 0.935 & 0.267 \\
\hline $11 \mathrm{H}-2,31$ & 89.11 & 1.8 & 142 & 3.51 & 183 & 27.4 & 10.0 & 61.2 & 11.2 & 28.1 & 4.23 & 21. & 5.42 & 1.75 & 7.96 & 1.240 & 7.80 & 1.51 & 4.48 & 0.515 & 0.466 & 0.144 \\
\hline $13 \mathrm{H}-6,35$ & 114.15 & 2.25 & 123 & 1.36 & 158 & 26.5 & 8.9 & 44.8 & 9.8 & 23.3 & 3.64 & 18 & 5.21 & 1.79 & 7. & 1.090 & 7.43 & 1.45 & 4.13 & 0.554 & 0.597 & 0.177 \\
\hline $\begin{array}{l}14 \mathrm{H}-3,0 \\
140\end{array}$ & 118.8 & 2.3 & 123 & $\begin{array}{l}1.50 \\
1.69\end{array}$ & $\begin{array}{l}108 \\
170\end{array}$ & 24.7 & $\begin{array}{l}8.9 \\
8.4\end{array}$ & $\begin{array}{l}43.0 \\
43.6\end{array}$ & $\begin{array}{l}9.0 \\
9.9\end{array}$ & 24.3 & 3.83 & 18 & 4.13 & 1. & 6. & 1.0 & $\begin{array}{l}7.43 \\
7.26\end{array}$ & 1. & 4.41 & 0.487 & 0.581 & 0.127 \\
\hline $14 \mathrm{H}-3$, & $\begin{array}{l}118.8 \\
118.8\end{array}$ & 2.3 & $\begin{array}{l}125 \\
152\end{array}$ & $\begin{array}{l}1.09 \\
4.29\end{array}$ & 200 & 27.5 & $\begin{array}{r}8.4 \\
11.6\end{array}$ & $\begin{array}{l}4.0 \\
69.7\end{array}$ & 12.5 & 30.5 & $\begin{array}{l}3.83 \\
4.45\end{array}$ & 21 & $\begin{array}{l}4.13 \\
5.34\end{array}$ & 2. & $\begin{array}{l}0.13 \\
6.90\end{array}$ & 1.3 & $\begin{array}{l}1.20 \\
7.34\end{array}$ & 1. & 4. & $\begin{array}{l}0.481 \\
0.651\end{array}$ & $\begin{array}{l}0.081 \\
0.549\end{array}$ & $\begin{array}{l}0.260 \\
0.260\end{array}$ \\
\hline 18H-CC, 14 & $\begin{array}{l}11.8 \\
161.8\end{array}$ & 2.5 & $\begin{array}{l}132 \\
287\end{array}$ & $\begin{array}{r}4.29 \\
27.90\end{array}$ & 39 & 40.5 & $\begin{array}{l}1.0 \\
77.7\end{array}$ & $\begin{array}{r}0.1 \\
431.0\end{array}$ & 53.2 & 112 & 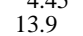 & 60 & $\begin{array}{l}5.34 \\
10.7\end{array}$ & 3.70 & $\begin{array}{r}0.90 \\
15.50\end{array}$ & $\begin{array}{l}1.020 \\
1.950\end{array}$ & $\begin{array}{l}10.34 \\
10.70\end{array}$ & 2. & 5.14 & $\begin{array}{l}0.031 \\
0.731\end{array}$ & 0.773 & $\begin{array}{l}0.600 \\
0.661\end{array}$ \\
\hline $20 \mathrm{H}-3,9$ & 172.89 & 2.52 & 105 & 7.98 & 18 & 2 & 6.9 & 125.0 & 9.1 & 20.9 & 2.61 & 11 & 3.53 & 1. & 4. & 0.814 & 4.78 & 0. & 2. & 0.382 & 0.376 & 0.218 \\
\hline $21 \mathrm{H}-2,10$ & 184.09 & 2. & 355 & 11.70 & 16 & 5 & 31 & 152 & 31.9 & 72.7 & 10 & 46 & 10 & 3. & 18. & 2380 & 15. & . & & 1.320 & 1.250 & 0.567 \\
\hline & & 2. & 323 & 10 & 181 & 4. & 25 & 13 & 25.5 & 56 & 7. & 30 & 7. & 2. & 11 & 2.010 & 10 & & 5 & 0. & 0.704 & 0.229 \\
\hline 14 & $\begin{array}{l}104 \\
205\end{array}$ & 2.6 & 218 & 9 & $\begin{array}{l}101 \\
169\end{array}$ & 37 & 16 & 9 & 18 & 45 & 6. & 30 & 7.8 - & & 11. & & 9. & & & & 0.913 & 0.325 \\
\hline 5,14 & 205.94 & 2.6 & 231 & 20.90 & 185 & 38 & 19.2 & 141.0 & 22 & 53.3 & 7.13 & 29 & 6.5 & 2. & 9. & 1.4 & 9.38 & 1.91 & 4.29 & 0.821 & 0.733 & 0.428 \\
\hline $23 X-5,67$ & 206.47 & 2.63 & 143 & 2.94 & 132 & 32.1 & 13.1 & 63.6 & 12.1 & 28.6 & 4.1 & 17.7 & 4.44 & 1.44 & 6.42 & 1.050 & 7.05 & 1.35 & 4.05 & 0.622 & 0.727 & 0.124 \\
\hline $23 X-5,67$ & 206.47 & 2.63 & 139 & 3.76 & 131 & 31.5 & 15.2 & 62.2 & 11.0 & 25 & 3.36 & 17.8 & 4.02 & 1.53 & 6.79 & 1.060 & 6.77 & 1.40 & 4.24 & 0.670 & 0.679 & 0.162 \\
\hline $23 \mathrm{X}-5,122$ & 207.12 & 2.65 & 227 & 6.90 & 187 & 38.8 & 27.3 & 96.2 & 19.5 & 46 & 6.14 & 29.4 & 6.14 & 2.57 & 10.10 & 1.440 & 8.83 & 1.77 & 4.62 & 0.765 & 0.758 & 0.226 \\
\hline
\end{tabular}




\begin{tabular}{|c|c|c|c|c|c|c|c|c|c|c|c|c|c|c|c|c|c|c|c|c|c|c|}
\hline $\begin{array}{l}\text { Core, section } \\
\text { interval }(\mathrm{cm})\end{array}$ & $\begin{array}{l}\text { Depth } \\
\text { (mbsf) }\end{array}$ & $\begin{array}{l}\text { Age } \\
\text { (Ma) }\end{array}$ & $\begin{array}{c}\mathrm{Zr} \\
(\mathrm{ppm})\end{array}$ & $\begin{array}{c}\mathrm{Rb} \\
(\mathrm{ppm})\end{array}$ & $\begin{array}{c}\mathrm{Sr} \\
(\mathrm{ppm})\end{array}$ & $\begin{array}{c}\mathrm{Y} \\
(\mathrm{ppm})\end{array}$ & $\begin{array}{c}\mathrm{Nb} \\
(\mathrm{ppm})\end{array}$ & $\begin{array}{c}\mathrm{Ba} \\
(\mathrm{ppm})\end{array}$ & $\begin{array}{c}\mathrm{La} \\
(\mathrm{ppm})\end{array}$ & $\begin{array}{c}\mathrm{Ce} \\
(\mathrm{ppm})\end{array}$ & $\begin{array}{c}\mathrm{Pr} \\
(\mathrm{ppm})\end{array}$ & $\begin{array}{c}\mathrm{Nd} \\
(\mathrm{ppm})\end{array}$ & $\begin{array}{c}\mathrm{Sm} \\
(\mathrm{ppm})\end{array}$ & $\begin{array}{c}\mathrm{Eu} \\
(\mathrm{ppm})\end{array}$ & $\begin{array}{c}\mathrm{Gd} \\
(\mathrm{ppm})\end{array}$ & $\begin{array}{c}\mathrm{Tb} \\
(\mathrm{ppm})\end{array}$ & $\begin{array}{c}\text { Dy } \\
(\mathrm{ppm})\end{array}$ & $\begin{array}{c}\text { Ho } \\
(\mathrm{ppm})\end{array}$ & $\begin{array}{c}\mathrm{Er} \\
(\mathrm{ppm})\end{array}$ & $\begin{array}{c}\mathrm{Tm} \\
(\mathrm{ppm})\end{array}$ & $\begin{array}{c}\mathrm{Lu} \\
(\mathrm{ppm})\end{array}$ & $\underset{(\mathrm{ppm})}{\mathrm{U}}$ \\
\hline $23 X-5,122$ & 207.12 & 2.65 & 132 & 2.20 & 204 & 23.4 & 12.3 & 56.1 & 10.7 & 26.4 & 4.02 & 16.3 & 4.2 & 1.42 & 4.81 & 0.876 & 5.51 & 1.20 & 2.94 & 0.441 & 0.503 & 0.210 \\
\hline $24 \mathrm{X}-3,128$ & 212.98 & 2.7 & 125 & 3.17 & 154 & 25.2 & 11.4 & 44.7 & 8.4 & 21.6 & 3.12 & 14.0 & 4.48 & 1.33 & 5.91 & 0.845 & 6.25 & 1.15 & 3.37 & 0.439 & 0.443 & 0.191 \\
\hline $24 \mathrm{X}-3,128$ & 212.98 & 2.7 & 220 & 4.29 & 183 & 42.9 & 19.2 & 95.0 & 17.3 & 42.5 & 5.76 & 27.4 & 7.25 & 2.64 & 8.33 & 1.530 & 9.82 & 2.01 & 4.31 & 0.934 & 0.830 & 0.151 \\
\hline $25 \mathrm{X}-4,109$ & 223.19 & 2.75 & 78.6 & 1.36 & 78.3 & 26.5 & 5.9 & 31.9 & 4.4 & 11.7 & 1.72 & 8.8 & 3.24 & 1.09 & 4.69 & 0.912 & 5.43 & 1.25 & 3.17 & 0.599 & 0.507 & 0.061 \\
\hline $25 \mathrm{X}-7,4$ & 226.64 & 2.8 & 561 & 16.60 & 174 & 50.9 & 36.6 & 176.0 & 31.5 & 68.7 & 9.11 & 44.8 & 7.97 & 2.95 & 8.24 & 2.010 & 10.80 & 2.52 & 4.60 & 1.070 & 0.608 & 0.736 \\
\hline $25 X-7,4$ & 226.64 & 2.8 & 357 & 12.00 & 173 & 45.0 & 27.1 & 145.0 & 23.9 & 61.7 & 8.06 & 31.7 & 7.96 & 2.67 & 10.30 & 1.790 & $\begin{array}{l}9.60 \\
9.69\end{array}$ & 2.44 & 5.83 & 0.838 & 0.697 & 0.439 \\
\hline $25 \mathrm{X}-7,4$ & 226.64 & 2.8 & 291 & 5.69 & 170 & 43.0 & 23.5 & 118.0 & 22.5 & 53.1 & 7.03 & 33. & 8.06 & 2.43 & 10.20 & 1.650 & 8.84 & 2.04 & 5.41 & 1.000 & 0.785 & 0.450 \\
\hline $33 \mathrm{X}-2,59$ & 290.49 & 3.2 & 205 & 7.30 & 194 & 34.0 & 19.9 & 98.0 & 15.4 & 36.9 & 5.1 & 23. & 5.1 & 2.1 & 7.43 & 1.34 & 8.04 & 1. & 4.4 & 0.687 & 0.579 & 0.221 \\
\hline $33 \mathrm{X}-2,59$ & 290.49 & 3.2 & 145 & 6.07 & 151 & 33.0 & 17.3 & 101.0 & 12.1 & 28.6 & 3.73 & 16. & 5.18 & 1.4 & 6.28 & 1.070 & $\begin{array}{l}0.04 \\
7.33\end{array}$ & 1.4 & 4.10 & 9 & 0.715 & 0.154 \\
\hline $33 \mathrm{X}-2,59$ & 290.49 & 3.2 & 255 & 8.61 & 235 & 39.3 & 29.4 & 119.0 & 22.6 & 52.4 & 7.26 & 34.0 & 7.49 & 2.55 & 9.55 & 1.620 & 9.27 & 1.82 & 4.81 & 0.710 & 0.621 & 0.430 \\
\hline \multicolumn{23}{|l|}{$152-9$} \\
\hline $39 \mathrm{R}-4,14$ & 652.54 & 13 & 121 & 73.10 & 41.9 & 20.1 & 17.2 & 470.0 & 28.8 & 50.9 & 5.12 & 15.3 & 2.89 & 1.69 & 4.70 & 0.664 & 3.76 & 0.91 & 2.88 & 0.224 & 0.502 & 2.340 \\
\hline $39 \mathrm{R}-4,14$ & 652.54 & 13 & 458 & 31.80 & 42.3 & 68.6 & 34.7 & 243.0 & 36.0 & 77.1 & 9.48 & 41.0 & 9.95 & 2.50 & 14.00 & 2.430 & 14.00 & 3.42 & 9.49 & 1.710 & 1.710 & 1.090 \\
\hline $40 \mathrm{R}-2,36$ & 659.36 & 13.5 & 514 & 42.40 & 70.1 & 55.9 & 43.7 & 323.0 & 39.4 & 78.4 & 9.47 & 35.0 & 7.58 & 2.65 & 13.10 & 1.960 & 1.23 & 2. & 8.12 & 1.250 & 1.440 & 1.550 \\
\hline $40 \mathrm{R}-2,36$ & 659.36 & 13.5 & 512 & 40.70 & 70.3 & 58.5 & 42.2 & 313.0 & 41.1 & 80.1 & 9.27 & 40 & 7.73 & 1 . & 11.30 & 1.980 & 12.80 & 2. & 8.41 & & 1.450 & 2.190 \\
\hline $40 \mathrm{R}-2,36$ & 659.36 & 13.5 & 481 & 37.60 & 68.7 & 56.4 & 39.8 & 305.0 & 41.4 & 83.4 & 9.74 & 39 & 9.26 & 2. & 12.10 & 2.060 & 12.30 & 2. & 7.58 & 1.200 & 1.150 & 1.360 \\
\hline $40 \mathrm{R}-2,36$ & 659.36 & 13.5 & 545 & 45.50 & 56.6 & 62.2 & 49.6 & 341.0 & $\begin{array}{l}41.4 \\
45.7\end{array}$ & 90.7 & 9.22 & 36. & 8.89 & 2.21 & 13.30 & 2.250 & 13.90 & 3. & 8.64 & 1.520 & 1.770 & 2.010 \\
\hline 40R-3, 114 & 660.86 & 13.5 & 496 & 42.00 & 54.1 & 64.6 & 46.6 & 337.0 & 44.8 & 89.9 & 10.7 & 44. & 9.37 & 2.41 & 12.70 & 2.230 & 15.30 & 3. & 10.40 & 1.450 & 1.820 & 2.450 \\
\hline$-3,114$ & 660.86 & 13.5 & 488 & 37.80 & 58.8 & 51.9 & 37.0 & 272.0 & 37.3 & 69.4 & 8.57 & 33. & 6.99 & 1.59 & 8.75 & 1.600 & 9.49 & 1.8 & 6.23 & 40 & 1.150 & 1.200 \\
\hline $40 \mathrm{R}-3,114$ & 660.86 & 13.5 & 231 & 52.50 & 44.9 & 65.7 & 59.0 & 404.0 & 53.0 & 114 & 12.9 & 54.9 & 12.3 & 2.95 & 18.00 & 2.230 & 15.70 & 2.85 & 8.49 & 1.370 & 1.460 & 1.860 \\
\hline $88 \mathrm{R}-1,42$ & 1118.22 & 42 & 1020 & 301.0 & 232 & 20.1 & 176.0 & 249.0 & 20.8 & 63.9 & 4.13 & 17.6 & 3.54 & 1.11 & 11.30 & 1.250 & 6.88 & 1.38 & 4.13 & 0.679 & 0.841 & 0.789 \\
\hline $96 \mathrm{R}-1,29$ & 1185.39 & 51 & 68.5 & 169.0 & 12.1 & 0.8 & 1.6 & 5.5 & 1.2 & 2.81 & 0.32 & 1.7 & 0.41 & 0.09 & 0.46 & 0.038 & 0.17 & 0.04 & 0.11 & 0.009 & 0.028 & 0.022 \\
\hline $100 \mathrm{R}-2,27$ & 1225.47 & 52 & 41.7 & 45.00 & 50.6 & 6.6 & 0.3 & 10.9 & 0.1 & 0.57 & 0.17 & 1.1 & 0.4 & 0.19 & 0.92 & 0.278 & 2.33 & 0.40 & 1.61 & 0.295 & 0.439 & 0.000 \\
\hline $100 \mathrm{R}-3,82$ & 1227.52 & 52 & 57.1 & 19.70 & 6.24 & 0.8 & 1.0 & 2.6 & 0.1 & 0.54 & 0.12 & 0.6 & 0.17 & 0.0 & 0.29 & 0.073 & 0.19 & 0.07 & 0.15 & 0.013 & 0.007 & 0.000 \\
\hline $100 \mathrm{R}-3,82$ & 1227.52 & 52 & 99.1 & 18.00 & 20.6 & 30.0 & 4.3 & 10.6 & 1.3 & 4.49 & 0.64 & 4.0 & 1.8 & 0.75 & 3.54 & 0.833 & 5.62 & 1.40 & 4.06 & 0.714 & 0.792 & 0.000 \\
\hline \multicolumn{23}{|l|}{ 152-919B- } \\
\hline & 10.26 & 0.1 & 19 & 5. & 174 & 35 & 16.8 & 82 & 15.1 & 36. & 5.0 & 24. & 6.5 & 2.07 & 8.1 & 1.170 & 8.31 & 1.69 & 4.14 & 0.719 & 0.506 & 0.238 \\
\hline & 10 & 0. & & & 22 & & 31 & 132 & & 58 & 7. & 36 & 8. & 2.4 & 12. & 1.6 & 9.1 & 1. & 5.07 & 0.712 & 0.662 & 0.478 \\
\hline & & & & & 22 & & 31 & 131 & & 57 & & & & & 10 . & & 8. & & & & & 0.486 \\
\hline $2 \mathrm{H}-1,108$ & 10.26 & 0.15 & 216 & 5.84 & 175 & 39.3 & 22.4 & 94.2 & 17.3 & 43 & 5.49 & 26.8 & $\begin{array}{l}6.70 \\
6.98\end{array}$ & 1.85 & 9.75 & 1.480 & 7.70 & 1.90 & 5.38 & 0.820 & 0.710 & 0.346 \\
\hline \multicolumn{23}{|l|}{ 152-919A- } \\
\hline & 21.35 & 0.3 & 197 & 5.93 & 172 & 35.0 & 17.2 & 80.4 & 14.8 & 37.9 & 4.88 & 24.6 & 6.59 & 1.93 & 9.35 & 1.200 & 8.53 & 1.56 & 4.77 & 0.682 & 0.729 & 0.169 \\
\hline & 3 & & 78.3 & 3.2 & 118 & 21 & 7.8 & 64.4 & 7.2 & 16.6 & 2. & 11 & 2. & 1. & 4. & 0.6 & 4.75 & & 2.89 & & 0.410 & 0.203 \\
\hline $7 \mathrm{H}-4,135$ & 61.35 & 0.87 & 260 & 7.39 & 212 & 35.1 & 28.4 & 124.0 & 24.4 & 57.4 & 7.49 & 3. & 7.9 & 2. & 8. & 1.5 & 7.85 & 1. & 4.50 & 0.586 & 0.427 & 0.231 \\
\hline $7 \mathrm{H}-4,135$ & 61.35 & 0.87 & 265 & 7.55 & 217 & 35.8 & 29.0 & 126.0 & 24.9 & 58.6 & 7.65 & 33.0 & 7.69 & 2.44 & 9.42 & 1.620 & 8.18 & 1.41 & 4.62 & 0.606 & 0.449 & 0.236 \\
\hline $7 \mathrm{H}-4,135$ & 61.35 & 0.87 & 284 & 6.35 & 230 & 37.6 & 34.1 & 143.0 & 27.4 & 56.4 & 8.11 & 38.3 & 8.18 & 2.58 & 10.20 & 1.400 & 8.57 & 1.29 & 3.85 & 0.541 & 0.750 & 0.469 \\
\hline \multicolumn{23}{|l|}{ 152-919B- } \\
\hline & 103.65 & 1.47 & 80.9 & 8.22 & 120 & 20. & 8.3 & 88.5 & 8. & 17.6 & 2.35 & 10 & 3.2 & 0.8 & 3.52 & 0.52 & 3.40 & 0.7 & 2.51 & 0.420 & 0.379 & 0.082 \\
\hline & & & & & 2 & & 38 & 15 & 30 & & & & & & 12 & & 9. & & & & 0.753 & 0.420 \\
\hline & & 1. & & & 18 & 4 & 23 & 121 & & 51 & 7. & & 7. & & 10. & 1.6 & 10. & 1. & 5.33 & & 0.623 & 0.464 \\
\hline & & 1.69 & 221 & 6. & 150 & 36 & 17.1 & 92 & 16 & 40 & 5.2 & 24 & 6.8 & 1. & 10. & 1.3 & 8. & 1. & 3.80 & 0.529 & 0.621 & 0.311 \\
\hline & 118.93 & 1.69 & 217 & 6. & 147 & 36.1 & 16.8 & 90 & 16 & 39.6 & 5. & 24 & 6. & 1. & 9. & 1.280 & 7.97 & 1. & 3.69 & 0.512 & 0.597 & 0.305 \\
\hline & 126.87 & 1.8 & 241 & 7.06 & 184 & 38.9 & 19.3 & 98.0 & 18 & 44.4 & 6.32 & 28 & 7.94 & 2. & 8. & 1.440 & 8.70 & 1. & 4.99 & 0.725 & 0.625 & 0.521 \\
\hline $6 \mathrm{H}-6,87$ & 126.87 & 1.8 & 241 & 7.06 & 184 & 38.9 & 19.3 & 98.0 & 18.6 & 44.4 & 6.32 & 28.2 & 7.94 & 2.64 & 8.95 & 1.440 & 8.70 & 1.86 & 4.99 & 0.725 & 0.625 & 0.521 \\
\hline $7 \mathrm{H}-1,37$ & 128.37 & 1.8 & 275 & 8.17 & 229 & 34.7 & 33.1 & 136.0 & 25.6 & 59.8 & 7.53 & 33.6 & 8.66 & 2.10 & 11.70 & 1.460 & 8.37 & 1.45 & 5.11 & 0.715 & 0.422 & 0.383 \\
\hline
\end{tabular}


Table 3. Representative analysis of a basaltic glass shard from Sample $152-918 \mathrm{~A}-8 \mathrm{H}-2,34 \mathrm{~cm}$, showing uncertainties for typical chemical composition.

\begin{tabular}{ccc}
\hline Element & $\begin{array}{c}\text { Concentration } \\
(\mathrm{ppm})\end{array}$ & \% Error \\
\hline $\mathrm{Rb}$ & 6.4 & 2.98 \\
$\mathrm{Sr}$ & 203 & 0.52 \\
$\mathrm{Y}$ & 41.3 & 0.94 \\
$\mathrm{Zr}$ & 251 & 0.67 \\
$\mathrm{Nb}$ & 25.2 & 2.00 \\
$\mathrm{Ba}$ & 96.9 & 1.27 \\
$\mathrm{La}$ & 18.6 & 2.33 \\
$\mathrm{Ce}$ & 45.8 & 1.57 \\
$\mathrm{Pr}$ & 5.97 & 3.88 \\
$\mathrm{Nd}$ & 30 & 4.93 \\
$\mathrm{Sm}$ & 6.83 & 9.33 \\
$\mathrm{Eu}$ & 2.72 & 5.14 \\
$\mathrm{Gd}$ & 9.95 & 4.23 \\
$\mathrm{~Tb}$ & 1.69 & 4.77 \\
$\mathrm{Dy}$ & 10 & 4.78 \\
$\mathrm{Ho}$ & 1.78 & 5.42 \\
$\mathrm{Er}$ & 5.14 & 6.64 \\
$\mathrm{Tm}$ & 0.675 & 8.61 \\
$\mathrm{Lu}$ & 0.556 & 9.49 \\
$\mathrm{U}$ & 0.28 & 31.60 \\
\hline
\end{tabular}

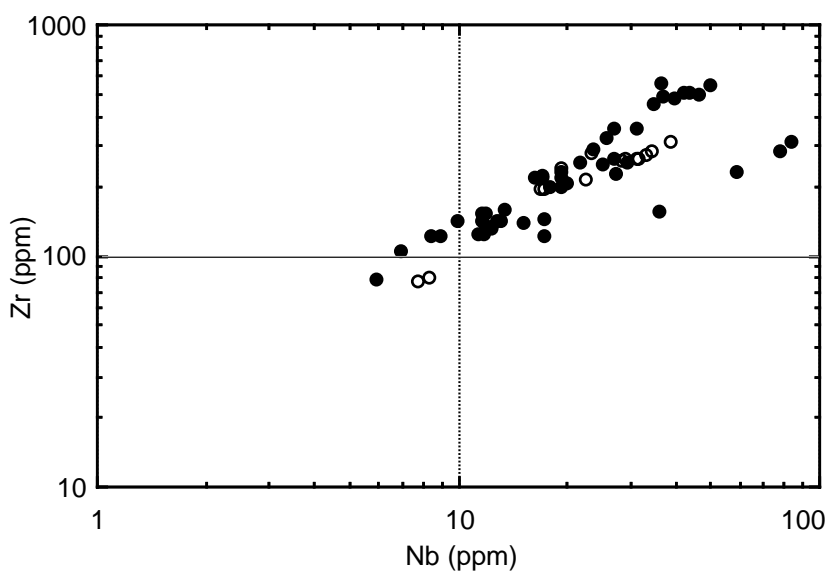

Figure 6. Variations in the concentrations of $\mathrm{Zr}$ with $\mathrm{Nb}$ contents from basaltic glass grains at Sites 918 and 919. Note the correlation and inferred high analytical precision at all measured concentrations. Site $918=$ solid circles and Site $919=$ open circles.

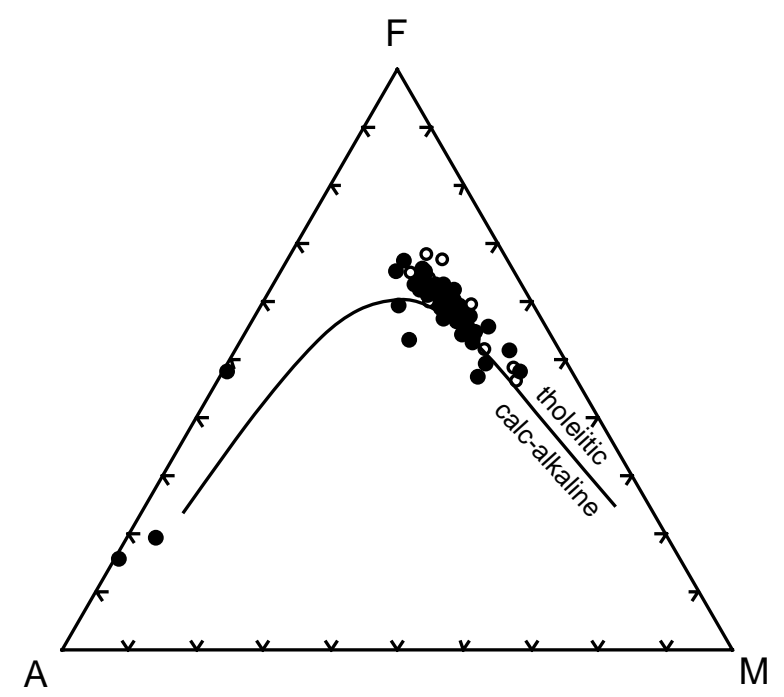

Figure 7. Triangular alkali/iron/magnesium (AFM) diagram showing the vast majority of glass grains from Sites 918 (solid circles) and 919 (open circles) falling within the tholeiite field, as defined by Irvine and Baragar (1971).
MORB (N-MORB), which typically show relative depletion in these elements compared to the less incompatible elements on the right of the diagram (Fig. 8A). A flat or incompatible enriched trace element pattern on the spider diagram is seen for Icelandic basalts and may be produced by either extracting a small degree $(<1 \%)$ of partial melt from a normal asthenospheric source, or by large degrees of melting of a less depleted source. Figure 8A shows a basalt from the modern volcano at Krafla in the eastern rift zone of Iceland (Hemond et al., 1993), whose form can be seen to be similar to that of more depleted Pleistocene ashes at Site 918. This similarity of trace element chemistry supports the Icelandic origin of the ashes. Since tholeiites are generated by moderate to large degrees of partial melting, the incompatible-enriched characteristics suggest that the drill core ashes and modern Icelandic lavas are derived from a plume mantle source.

The middle Miocene spider diagrams in Figure $8 \mathrm{C}$ show greater enrichment for most elements compared to primitive mantle than the younger examples shown. This is especially true for the most incompatible elements. This does not reflect more incompatible-enriched volcanism at that time, but instead a concentration of incompatible elements by fractional crystallization during the formation of these rhyolites. All younger glasses plotted were from basaltic shards and did not show the effects of this process. Fractional crystallization also explains the large degrees of depletion seen in $\mathrm{Sr}, \mathrm{P}$, and Ti relative to the other elements. Sr, P, and Ti are removed respectively by the crystallization of plagioclase, apatite, and spinel prior to eruption.

The oldest Eocene glasses from Site 918 show different trace patterns that are noticeably flatter and incompatible-element depleted than the younger grains. On the left hand side of the diagram (Fig. $8 \mathrm{D})$, the stronger hydration of these grains is shown by the strong enrichment of water mobile elements. Sample 152-918D-100R-3, 82 $\mathrm{cm}$, in particular shows a clear depletion in the more incompatible elements compared to the less incompatible elements on the right of the diagram. The slope to the spider diagram is typical of lavas derived by melting from a depleted mantle source, and is similar to those plotted for the basalts on which these sediments lie.

\section{Rare Earth Elements}

Rare earth elements are often considered in isolation from the other trace elements and are shown arranged in order of increasing atomic number (Fig. 10). In this diagram, it is conventional to normalize concentrations against a chondritic source, with the values of Sun and McDonough (1989) being used in this study. It is noteworthy that in all the examples shown in Figure 10 the general slope of the curve shows an enrichment in light REE (LREE) relative to heavy REE (HREE). The relative HREE depletion may indicate melting in the presence of garnet, which preferentially retains the HREE. Again, the Miocene rhyolitic glasses shown in Figure 10C show greater enrichment relative to the normalizing standard for all elements, due to the effects of fractional crystallization. The relative Eu depletion is characteristic of plagioclase fractionation. Nonetheless, the generally incompatible nature of the REE means that while total concentrations rise during crystallization, the slope of the line remains largely unchanged. In the basaltic examples, we see that while the HREE show concentrations of $20 \times$ to $30 \times$ chondrite, the degree of LREE enrichment over HREE is variable over short time spans. The general relatively high incompatibility of the REE means that the slope on the REE diagram may be representative of the source mantle if the degree of partial melting remains constant. The REE diagrams suggest either short-term variability of the mantle source of the ashes or variation in the degree of melting.

\section{Temporal Evolution}

The temporal variability of elemental enrichment and depletion can be charted by plotting key element ratios against age for both Sites 918 and 919. Enrichment of incompatible elements in both the high field strength and REE can be modeled using $\mathrm{Zr} / \mathrm{Nb}$ and $\mathrm{Ho} / \mathrm{La}$, 
A

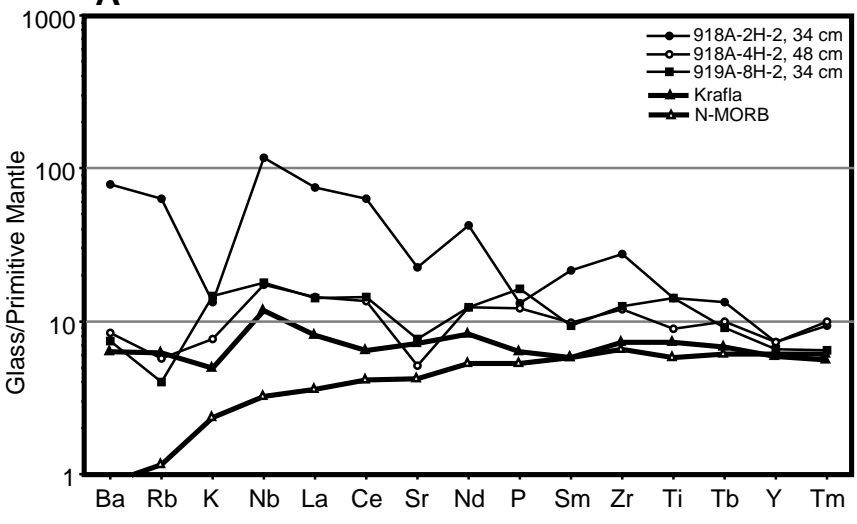

B

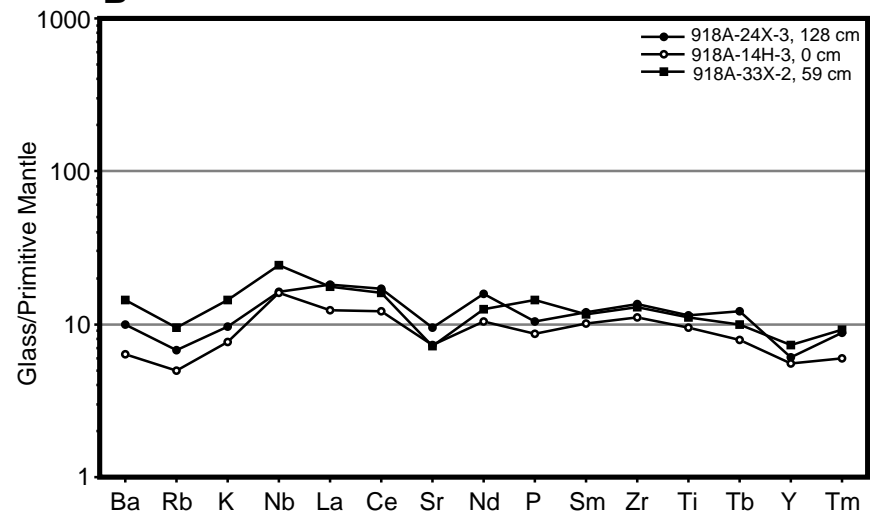

C

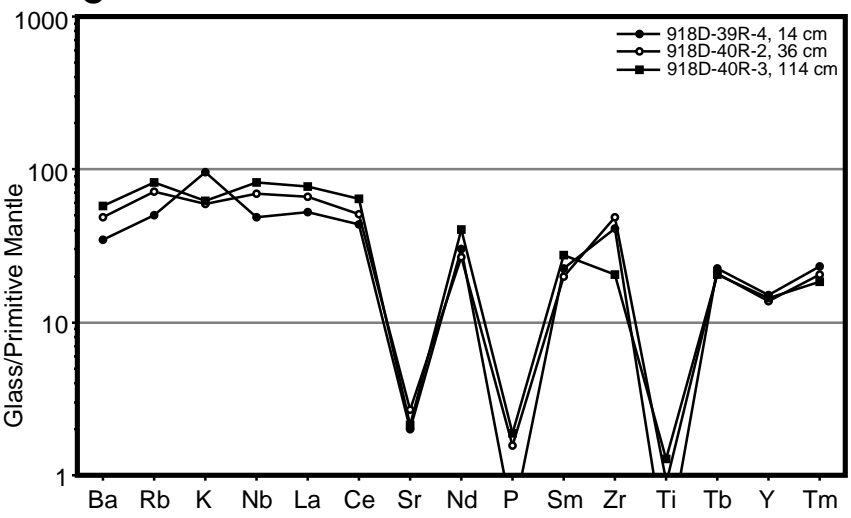

D

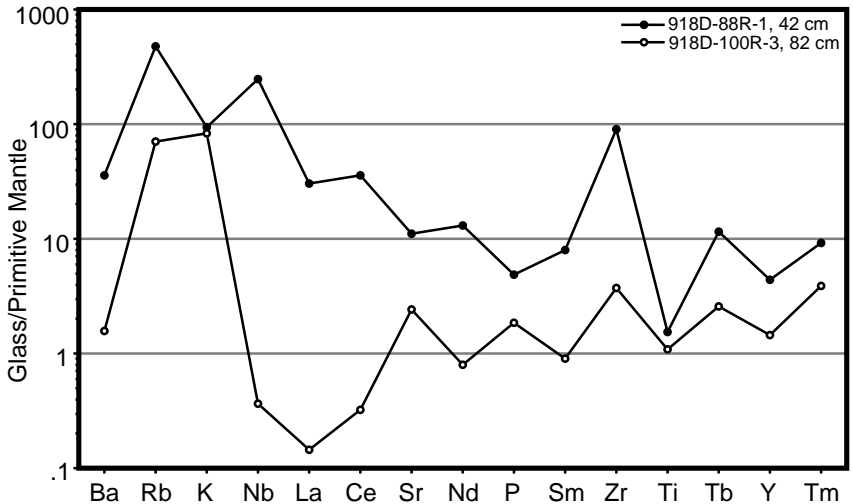

Figure 8. Spider diagrams of basaltic glass grains from (A) Pleistocene at Site 918, including N-MORB (Sun and McDonough, 1989) and a basalt from Krafla in the eastern rift zone of modern Iceland (Hemond et al., 1993); (B) Pliocene at Site 918; (C) Miocene at Site 918 ; and (D) Oligocene and Eocene at Site 918 (Thompson et al., 1983).

respectively. These ratios reflect source characteristics and the degree of partial melting, but are unaffected by fractional crystallization in basalts. Figure 11 shows the spread of values measured for the post $15 \mathrm{Ma}$ ashes. For comparison, the values for typical N-MORB and ocean island basalt (OIB) are shown, as well as the range for Icelandic basalts from the Tertiary lava pile and the neovolcanic zone (Hardarson and Fitton, 1994). It is clear that for the most part the composition of the ashes corresponds to that of the Icelandic Tertiary lava sequences and is broadly comparable with that of a typical OIB. However, a considerable number of analyses show higher $\mathrm{Zr} / \mathrm{Nb}$ than in Icelandic Tertiary basalts, although the range is not as large as in the neovolcanic zone.

Figure 12 shows the composition of Neogene ashes at Sites 918 and 919 compared with basaltic lavas from Iceland. In this diagram the value of Y used was recalculated from the Ho content because the analyzed Y/Ho values were consistently lower than the 27.7 reported by McDonough and Sun (1995). Since the ratio of these elements is very stable due to their very similar compatibility, the lower than expected $\mathrm{Y}$ values suggest a consistent analytical shortfall using the ion probe for reasons that are not apparent. The parallel lines on the diagram represent the limit of compositions of basalt from the Icelandic neovolcanic zone (Fitton, Saunders, et al., this volume). The + symbol represents the composition of primitive mantle (McDonough and Sun, 1995), which plots within the Iceland array. Depleted Icelandic basalt plots below and to the left of this point, but most Icelandic lavas plot above and to the right. N-MORB plot below the lower bound of the array because they are more depleted in $\mathrm{Nb}$ with respect to other incompatible elements than are even the most depleted basalts from Iceland.

Individual data points are shown for Tertiary basalt from East Iceland and for Pleistocene-Holocene basalt from the Snaefellsnes Pen- insula. The Tertiary basalt pile is composed of flows large enough to have escaped the axial rift zone and therefore avoid burial deep in the crust (Hardarson and Fitton, 1994). These large flows probably provide a good estimate of the average composition of magma erupted within the rift zone. The Snaefellsnes lavas, by contrast, were erupted off-axis. It is noteworthy that all the ash samples from Sites 918 and 919 plot within the Iceland array and that most overlap the field of Tertiary basalt. Three samples have higher $\mathrm{Nb} / \mathrm{Y}$ and resemble basalt from Snaefellsnes. No other neovolcanic rocks from Iceland plot in this part of the diagram. We conclude that the bulk of the recovered ashes were erupted from the Iceland rift zone, with two samples (152918A-2H-2, $34 \mathrm{~cm}$, and 152-918A-18H-CC, $14 \mathrm{~cm}$ ) probably erupted from an off-axis volcano, possibly Snaefelles itself.

\section{DISCUSSION}

The Neogene-Holocene ashes recovered from Sites 918 and 919 show a predominantly basaltic character over the past $3.5 \mathrm{~m}$.y. The Icelandic origin of this material is effectively demonstrated by their tholeiitic character, which rules out the alternative of Jan Mayen Island. The middle Miocene ashes that have survived diagenesis are also considered to be Icelandic on the basis of their low alkali content. The patchy nature of the record prior to $3.5 \mathrm{Ma}$ is presumably due to diagenetic alteration of volcanic glass to clay in older sediments, except in rare circumstances in which the grains were preserved by early cementation or by incorporation into impermeable layers. The record preserved is probably not a very complete one given the position of Sites 918 and 919 upwind of the source volcanoes in Iceland. This is thus not a good place to test models suggesting an episodicity to Icelandic volcanism. 

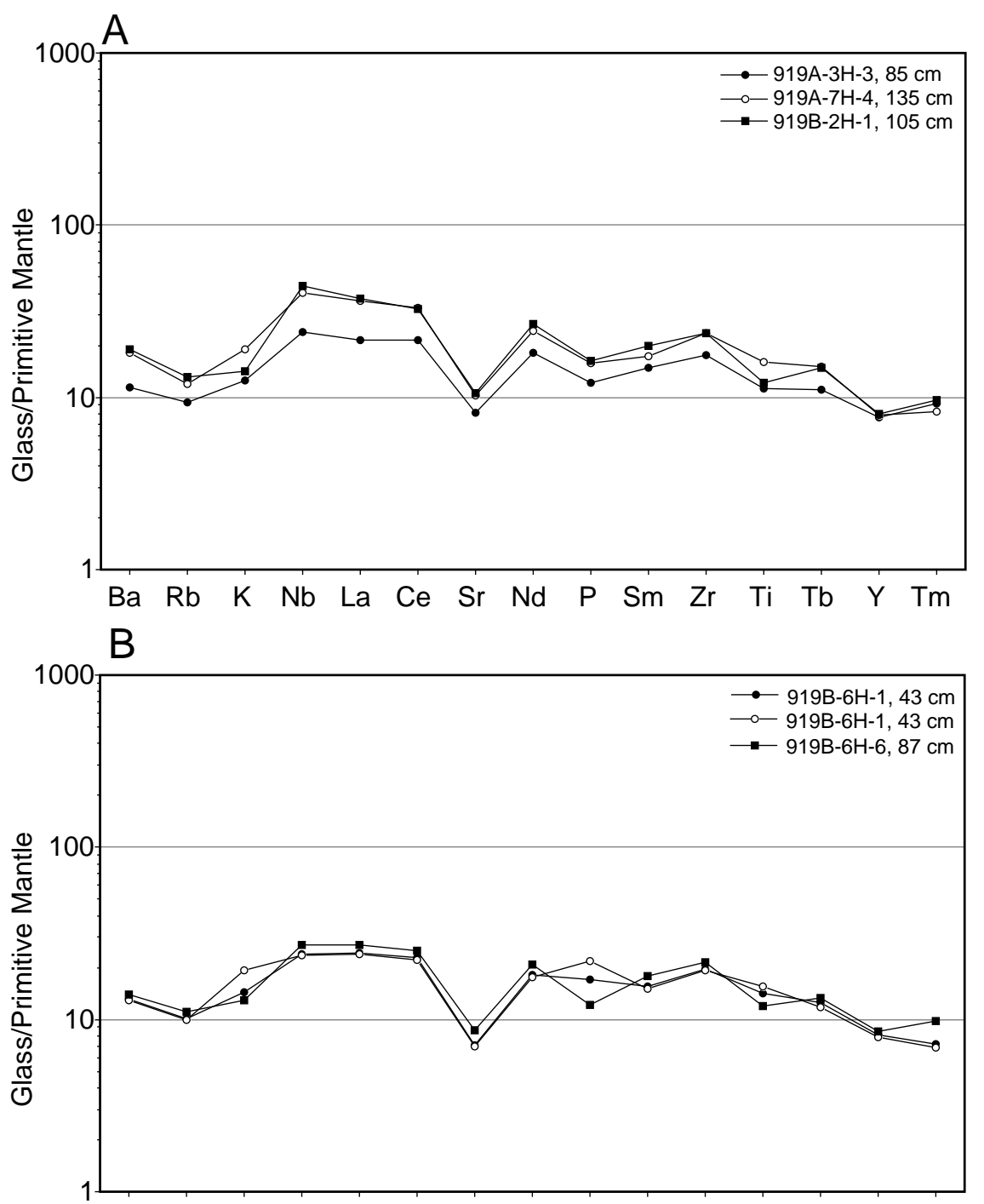

Figure 9. Spider diagrams of basaltic glass grains from $\begin{array}{lllllllllllllll}\mathrm{Ba} & \mathrm{Rb} & \mathrm{K} & \mathrm{Nb} & \mathrm{La} & \mathrm{Ce} & \mathrm{Sr} & \mathrm{Nd} & \mathrm{P} & \mathrm{Sm} & \mathrm{Zr} & \mathrm{Ti} & \mathrm{Tb} & \mathrm{Y} & \mathrm{Tm}\end{array}$ (A) Pleistocene at Site 919 and (B) Pliocene at Site 919.

In contrast to the East Greenland sediments, Bitschene et al. (1989) found a dominance of rhyolitic shards in Neogene-Holocene sediments on the Vøring Plateau. In this area Iceland was considered to be the principal source for the Neogene ashes. The acidic tephra were sourced from both Iceland (c.f. Walker, 1966) and Jan Mayen Island, and were distinguished by their alkali contents (Sylvester, 1978; Maaløe et al., 1986). In the case of Sites 918 and 919, the glasses are all tholeiitic and thus of a purely Icelandic origin. Volcanism in East Greenland is believed to have continued until the early Miocene (Gleadow and Brooks, 1979), but cannot have been the source for the Neogene ashes analyzed here.

The record from Sites 918 and 919 shows that there is no longterm variability in the relative depletion of Icelandic volcanism and that over short periods of time liquids of both more and less depleted character were being erupted. The variability in high field strength elements enrichment at approximately constant LREE enrichments indicates that the degree of partial melting is variable. The degree of variability in $\mathrm{Zr} / \mathrm{Nb}$ is not as high as seen in the modern neovolcanic zones (c.f. Nicholson, 1990; Furman et al., 1991; Elliot et al., 1991), but is more comparable to the variability found in the Tertiary lava pile (Hardarson and Fitton, 1994). This is compatible with the predictions of the Pálmason $(1981 ; 1986)$ spreading model, which would predict the deep burial of small volume flows erupted close to the rift axis. Exactly what the variability in the degree of enrichment represents is uncertain without isotopic data. Such variations in trace and rare earth element depletion could represent melt extraction from variably depleted parts of the upwelling asthenosphere, as Hemond et al. (1993) were able to demonstrate using $\mathrm{Sr}$ and $\mathrm{Nd}$ isotopes. However, given that melts are being extracted from a long column of asthenosphere at any one time and mixed to a variable degree with melts from other depths before eruption (McKenzie and Bickle, 1988), it is possible to generate the observed variability in depletion by erupting melts extracted from different depths, while keeping the general composition of the plume mantle constant. In this case the least depleted rocks would be derived from melts mixed from the length of the melting column. The more depleted rocks could represent melt extractions from just the higher parts of the column, from mantle that has already undergone melt extraction at lower levels. Elliot et al. (1991) have demonstrated such variability from lavas from the Reykjanes Peninsula and from Theistareykir in northern Iceland. However, the most depleted Icelandic lavas form small-volume flows confined to the rift axis. These will not be preserved in the observable part of the Tertiary lava pile, nor are they likely to contribute to the ashes studied here.

\section{ACKNOWLEDGMENTS}

PC would like to thank John Craven, Richard Hinton, and Stuart Kearns at the University of Edinburgh for their hospitality and pro- 

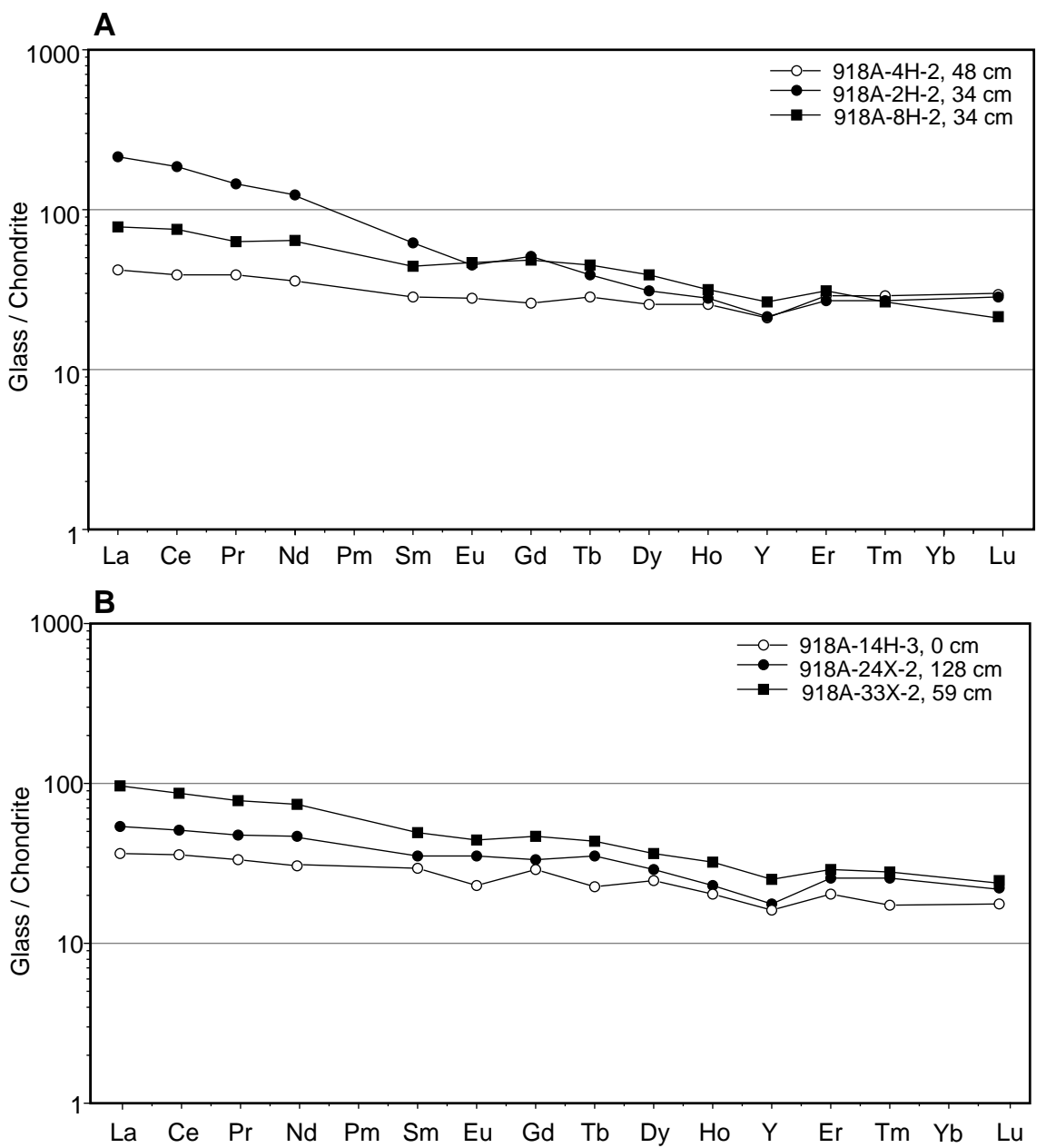

Figure 10. Chondrite normalized REE diagrams for basaltic grains from Site 918: (A) Pleistocene, (B) Pliocene, and (C) Miocene.

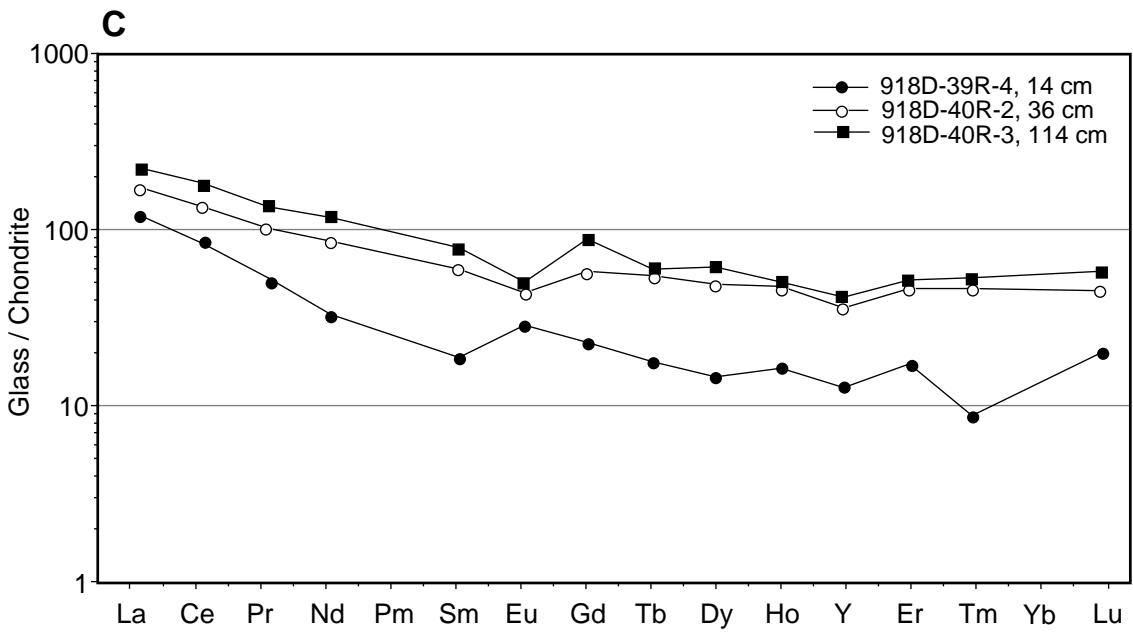

fessional advice during his visit to use the ion microprobe. The Ocean Drilling Program is thanked for providing both support in the sedimentology laboratory and computer equipment to write up this work. JOI/USSAC are thanked for their financial support of this project. Laura Dancer, Mohit Jain, and Joel Prellop are thanked for their help in the sample preparation.

\section{REFERENCES}

Bitschene, P.R., Schmincke, H.-U., and Viereck, L., 1989. Cenozoic ash layers on the Vøring Plateau (ODP Leg 104). In Eldholm, O., Thiede, J.,
Taylor, E., et al., Proc. ODP, Sci. Results, 104: College Station, TX (Ocean Drilling Program), 357-366.

Bouma, A.H., 1962. Sedimentology of Some Flysch Deposits: A Graphic Approach to Facies Interpretation: Amsterdam (Elsevier).

Burnham, C.W., and Jahns, R.H., 1962. A method of determining the solubility of water in silicate melts. Am. J. Sci., 260:721-745.

Cande, S.C., and Kent, D.V., 1992. A new geomagnetic polarity time scale for the Late Cretaceous and Cenozoic. J. Geophys. Res., 97:1391713951.

Clift, P.D., and Dixon, J.E., 1994. Variations in arc volcanism and sedimentation related to rifting of the Lau Basin (southwest Pacific). In Hawkins, J., Parson, L., Allan, J., et al., Proc. ODP, Sci. Results, 135: College Station, TX (Ocean Drilling Program), 23-49. 
Desprairies, A., Maury, R.C., Joron, J.-L., Bohn, M., and Tremblay, P., 1989. Distribution, chemical characteristics, and origin of ash layers from ODP Leg 104, Vøring Plateau, North Atlantic. In Eldholm, O., Thiede, J., Taylor, E., et al., Proc. ODP, Sci. Results, 104: College Station, TX (Ocean Drilling Program), 337-356.

Donn, W.L., and Ninokovich, D., 1980. Rate of Cenozoic explosive volcanism in the North Atlantic inferred from deep sea core. J. Geophys. Res., 85:5455-5460.

Elliot, T.R., Hawkesworth, C.J., and Grönvold, K., 1991. Dynamic melting of the Iceland plume. Nature, 351:201-206.

Furman, T., Frey, F.A., and Park, K.H., 1991. Chemical constraints on the petrogenesis of mildly alkaline lavas from Vestmannaeyjar, Iceland; the Eldfell (1973) and Surtsey (1963-1967) eruptions. Contrib. Mineral. Petrol., 109:19-37.

Garcia, M.O., and Hull, D.M., 1994. Turbidites from giant Hawaiian landslides: results from Ocean Drilling Program Site 842. Geology, 22:159162

Gleadow, A.J.W., and Brooks, C.K., 1979. Fission track dating, thermal histories and tectonics of igneous intrusions in East Greenland. Contrib. Mineral. Petrol., 71:45-60.

Hardarson, B.S., and Fitton, J.G., 1994. Geochemical variation in the Tertiary basalts of Iceland. Mineral. Mag., 58A:372-373.

Hart, S.R., Schilling, J.-G., and Powell, J.L., 1973. Basalts from Iceland and along the Reykjanes Ridge: Sr isotope geochemistry. Nature, 246:104107.

Hay, R.L., and Iijima, A., 1968. Nature and origin of palagonite tuffs of the Honolulu Group on Oahu, Hawaii. In Coats, R.R., Hay, R.L., and Anderson, C.A. (Eds.), Studies in Volcanology. Mem.-Geol. Soc. Am., 116:331-376.

Hemond, C., Arndt, N.T., Lichtenstein, U., Hoffman, A.W., Oskarsson, N., and Steintthorsson, S., 1993. The heterogeneous Iceland plume: Nd-Sr-O isotopes and trace element constraints. J. Geophys. Res., 98:1583315850 .

Hinton, R.W., and Upton, B.G.J., 1991. The chemistry of Zircon: variations within and between large crystals from syenite and alkali basalt xenoliths. Geochim. Cosmochim. Acta, 55:3287-3302.

Irvine, T.N., and Baragar, W.R.A., 1971. A guide to the chemical classification of the common volcanic rocks. Can. J. Earth Sci., 8:523-548.

Kennett, J.P., 1981. Marine tephrochronology. In Emiliani, C. (Ed.), The Sea (Vol. 7): The Oceanic Lithosphere: New York (Wiley), 1373-1436.

Klein, E.M., and Langmuir, C.H., 1987. Global correlations of ocean ridge basalt chemistry with axial depth and crustal thickness. J. Geophys. Res., 92:8089-8115.

Larsen, H.C., Saunders, A.D., Clift, P.D., et al., 1994. Proc. ODP, Init. Repts., 152: College Station, TX (Ocean Drilling Program).

Maaløe, S., Sorensen, I., and Hertogen, J., 1986. The trachybasaltic suite of Jan Mayen. J. Petrol., 27:439-466.

MacDonald, R., McGarvie, D.W., Pinkerton, H., Smith, R.L., and Palacz, Z.A., 1990. Petrogenetic evolution of the Torfajökull volcanic complex, Iceland, I. Relationship between the magma types. J. Petrol., 31:2:429459

McDonough, W.F., and Sun, S.-S., 1995. The composition of the Earth. Chem. Geol., 120:223-253.

McKenzie, D., and Bickle, M.J., 1988. The volume and composition of melt generated by extension of the lithosphere. J. Petrol., 29:625-679.

Mertz, D., Devey, C., Todt, W., Stoffers, P., and Hofmann, A., 1991. Sr-Nd$\mathrm{Pb}$ isotope evidence against plume-asthenosphere mixing north of Iceland. Earth Planet. Sci. Lett., 107:243-255.

Moberly, R., and Campbell, J.F., 1984. Hawaiian hotspot volcanism mainly during geomagnetic normal intervals. Geology, 12:459-63.

Neilson, C.H., and Sigurdsson, H., 1981. Quantitative methods for electron microprobe analysis of sodium in natural and synthetic glasses. Am. Mineral., 66:547-552.

Nicholson, H., 1990. The magmatic evolution of Krafla, NE Iceland [Ph.D. thesis]. Univ. of Edinburgh
Nicholson, H., Condomines, M., Fitton, J.G., Fallick, A.E., Grönvold, K., and Rogers, G., 1990. Geochemical and isotopic evidence for crustal assimilation beneath Krafla, Iceland. J. Petrol., 32:1005-1020.

Pálmason, G., 1981. Crustal rifting and related thermo-mechanical processes in the lithosphere beneath Iceland. Geol. Rundsch., 70:244-260.

, 1986. Model of crustal formation in Iceland, and application to submarine mid-ocean ridges. In Vogt, P.R., and Tucholke, B.E. (Eds.), The Western North Atlantic Region. Geol. Soc. Am., Geol. of North Am. Ser., M:87-98.

Pearce, J.A., 1983. The role of sub-continental lithosphere in magma genesis at destructive plate margin. In Hawkesworth, C.J., and Norry, M.J. (Eds.), Continental Basalts and Mantle Xenoliths: Nantwich (Shiva), 230-249.

Schilling, J.-G., 1973. Iceland mantle plume: geochemical study of Reykjanes Ridge. Nature, 242:565-571.

Schmincke, H.-U., Viereck, L.G., Griffin, B.J., and Pritchard, R.G., 1982. Volcaniclastic rocks of the Reydarfjordur Drill Hole, eastern Iceland. 1: Primary features. J. Geophys. Res., 87:6437-6458.

Shackleton, N.J., Backman, J., Zimmerman, H., Kent, D.V., Hall, M.A., Roberts, D.G., Schnitker, D., Baldauf, J.G., Desprairies, A., Homrighausen, R., Huddlestun, P., Keene, J.B., Kaltenback, A.J., Krumsiek, K.A.O., Morton, A.C., Murray, J.W., and Westberg-Smith, J., 1984. Oxygen isotope calibration of the onset of ice-rafting and history of glaciation in the North Atlantic region. Nature, 307:620-623.

Shipboard Scientific Party, 1994a. Site 917. In Larsen, H.C., Saunders, A.D., Clift, P.D., et al., Proc. ODP, Init. Repts., 152: College Station, TX (Ocean Drilling Program), 107-158.

1994b. Site 918. In Larsen, H.C., Saunders, A.D., Clift, P.D., et al., Proc. ODP, Init. Repts., 152: College Station, TX (Ocean Drilling Program), 177-256.

, 1994c. Site 919. In Larsen, H.C., Saunders, A.D., Clift, P.D., et al., Proc. ODP, Init. Repts., 152: College Station, TX (Ocean Drilling Program), 257-277.

Sigurdsson, H., and Loebner, B., 1981. Deep-sea record of Cenozoic explosive volcanism in the North Atlantic. In Self, S., and Sparks, R.S.J. (Eds.), Tephra Studies: Dordrecht (Reidel), 289-316.

Sun, S.-S., 1980. Lead isotopic study of young volcanic rocks from midocean ridges, ocean islands and island arcs. Philos. Trans. R. Soc. London A, 297:409-445.

Sun, S.-S., and McDonough, W.F., 1989. Chemical and isotopic systematics of oceanic basalts: implications for mantle composition and processes. In Saunders, A.D., and Norry, M.J. (Eds.), Magmatism in the Ocean Basins. Geol. Soc. Spec. Publ. London, 42:313-345.

Sylvester, A.G., 1978. Petrography of volcanic ashes in deep-sea cores near Jan-Mayen Island: Sites 338, 345-350, DSDP Leg 38. In Talwani, M., Udintsev, G., et al., Init. Repts. DSDP, Suppl. to 38, 39, 40, 41: Washington (U.S. Govt. Printing Office), 101-109.

Thompson, R.N., Morrison, M.A., Dickin, A.P., and Hendry, G.L., 1983. Continental flood basalts...arachnids rule OK? In Hawkesworth, C.J., and Norry, M.J. (Eds.), Continental Basalts and Mantle Xenoliths: Nantwich, UK (Shiva), 158-185.

Walker, G.P.L., 1966. Acid volcanic rocks in Iceland. Bull. Volcanol., 29:375-406.

Walker, G.P.L., and Croasdale, R., 1972. Characteristics of some basaltic pyroclastics. Bull. Volcanol., 35:303-317.

Zindler, A., Hart, S.R., Frey, F.A., and Jakobsson, S.P., 1979. Nd and Sr isotope ratios and REE abundances in Reykjanes Peninsula basalts: evidence for mantle heterogeneity beneath Iceland. Earth Planet. Sci. Lett., 45:249-262.

Date of initial receipt: 21 August 1995

Date of acceptance: 3 July 1996

Ms 152SR-203 

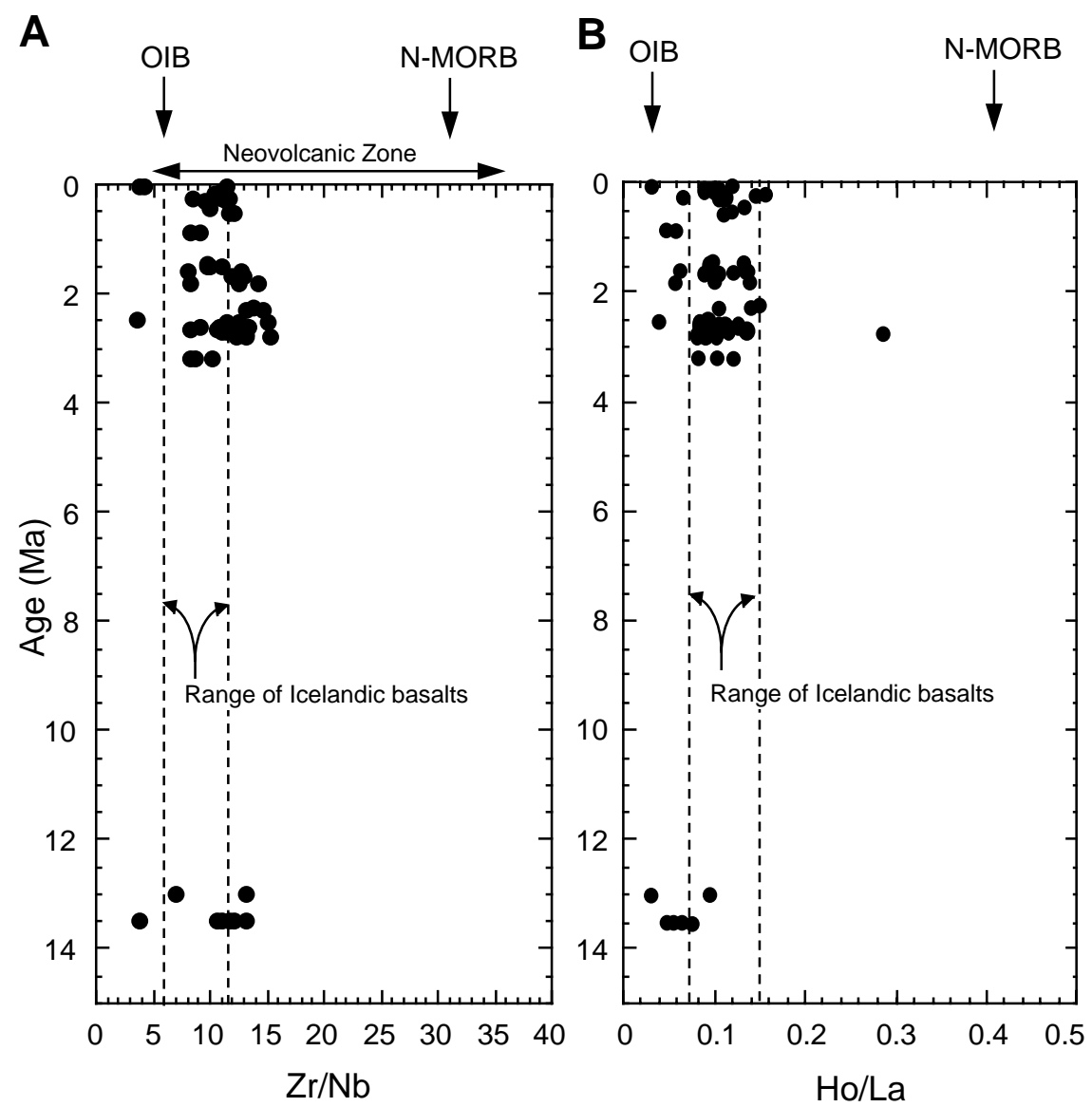

Figure 11. Diagram showing the variability in (A) $\mathrm{Zr} / \mathrm{Nb}$ and (B) $\mathrm{Ho} / \mathrm{La}$ with time at Sites 918 and 919 . Analyses with $\mathrm{MgO}>4 \%$ only. Icelandic values from Zindler et al. (1979), Nicholson (1990), MacDonald et al. (1990), Nicholson et al. (1991), and Hemond et al. (1993).

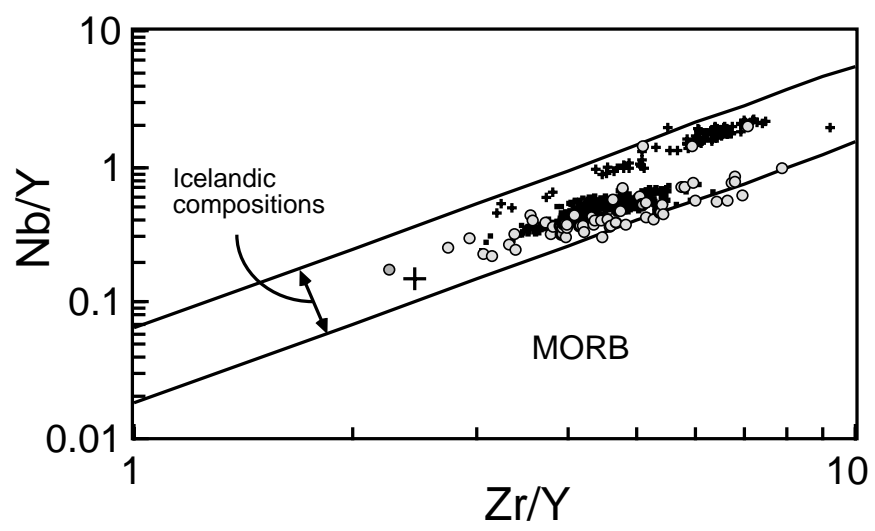

Figure 12. Variations in $\mathrm{Nb} / \mathrm{Y}$ vs. $\mathrm{Zr} / \mathrm{Y}$ (Fitton, Saunders, et al., this volume) showing the range of values for Neogene ashes from Leg 152 drill sites (gray circles), the Icelandic Tertiary lavas (small black squares), and the Snaefelles volcano of western Iceland (small crosses) for comparision. The two parallel lines represent the range of values known from the Icelandic system, within which the ashes fall. The large cross represents primitive mantle (Sun and McDonough, 1989). 